Ann. Biol. anim. Bioch. Biophys., I968, 8 (2), I47-193.

\title{
GUIDE POUR LA DISSECTION ET L'IDENTIFICATION DES PRINCIPAUX MUSCLES DE LA CARCASSE CHEZ LE MOUTON
}

\author{
M. BENÉVENT \\ avec la collaboration technique de C. BRESSOT \\ Laboratoire de Zootechnie, \\ Centre de Recherches agronomiques du Midi, \\ École nationale supérieure agronomique, 34 - Montpellier \\ Institut national de la Recherche agronomique
}

De nombreux groupes de chercheurs s'intéressent actuellement à différents aspects de la croissance des animaux domestiques, et ces études nécessitent souvent une dissection musculaire soit partielle, soit complète, des animaux en cause.

Différents traités d'anatomie peuvent être utilisés. Mais ils se rapportent le plus souvent, ainsi que les planches qui les illustrent, à la myologie des grands animaux (chevaux, bovins), les autres espèces n'y étant éventuellement mentionnées qu'à l'occasion des différences présentées par rapport à l'espèce type décrite (A. CHAUveau, S. Arloing, F. X. Lesbre, I903 ; L. Montane, E. Bourdelife, I9I7; S. Sisson, J. D. Grossman, I953 ; R. TAGaNd, R. BARone, I952). - Un travail ancien de CORNEVIN et LESBRE, (I892) souligne utilement les caractères myologiques différentiels du Mouton et de la Chèvre, sans toutefois décrire la dissection musculaire. Des éléments de myologie ovine peuvent cependant être trouvés dans un ouvrage beaucoup plus récent et spécialisé : The Anatomy of the sheep (N. D. S., MAY, I964).

Ces divers traités d'anatomie, souvent fort complets et détaillés, mais établis dans une autre optique en vue d'études vétérinaires, ne sont pas toujours d'une utilisation très commode pour celui qui recherche avant tout un moyen de reconnaissance efficace et rapide des principaux muscles.

Ayant eu à mesurer, à l'occasion de nos propres travaux, certaines difficultés découlant de cet état de faits, nous avons cru bien faire de présenter une série de planches schématisant les différentes étapes de la dissection musculaire d'une carcasse de mouton, afin de faciliter le travail des chercheurs qui auraient à s'intéresser à la myologie ovine.

Concernant cette espèce, une technique complète de dissection a d'ailleurs été décrite par P.-D. FouRIE (Ig62), mais les planches photographiques présentées à cette occasion manquent souvent de netteté et nous semblent insuffisantes pour assurer une reconnaissance rapide des principaux muscles et de leurs dispositions relatives. 
Notre travail se rapportant à la seule carcasse, les muscles de la tête n'y figurent pas, non plus que le diaphragme. En outre, nous nous sommes volontairement limités aux muscles principaux aisément séparables dans leur entier sans une prolongation excessive du temps de dissection.

En effet, les travaux relatifs à la croissance comportent généralement des mesures pondérales et il importe de limiter notamment les pertes par déshydratation. C'est pourquoi certains muscles doivent être prélevés en bloc (intercostaux). D'autres, sans intérêt particulier (muscles de la queue) ou inconstants (petit dentelé crânial) peuvent être négligés quant à leur identification et sont seulement pesés pour compléter l'évaluation de la masse musculaire totale. Ainsi se trouvent également exclus : muscles peauciers ; muscles de la queue ; intercostaux ; sus-costaux ; intertransversaires des lombes ; petit dentelé crânial (inconstant) ; transverse du thorax ; interépineux.

En vue d'éventuelles déterminations plus complètes, on pourra se reporter aux ouvrages d'anatomie cités en références bibliographiques, et que nous avons nousmêmes utilisés.

\section{MATÉRIEL ET MÉTHODE}

Toutes nos dissections ont été pratiquées sur des animaux de différents formats et de l'un ou l'autre sexe de la race ovine Mérinos d'Arles. Les schémas que nous présentons ont été réalisés à partir d'agneaux dont le poids vif était compris entre 20 et $3^{\circ} \mathrm{kg}$.

La préparation et le début de la dissection peuvent être conduits selon la technique indiquée par P.-D. FouRIE ( 1962 ) ou une technique voisine. L'animal est dépouillé, éviscéré, et les extrémités sont séparées de façon à obtenir la carcasse classique de boucherie. Après enlèvement du diaphragme, des reins et de leur enveloppe de gras interne, nous divisons alors la carcasse, de façon aussi précise que possible, en deux demi-carcasses pratiquement symétriques. Toutes nos planches, sauf celle où une présentation symétrique nous a parue nécessaire pour une meilleure compréhension (pl. 2), se rapportent à la demi-carcasse droite.

La face externe de celle-ci se présente d'abord entièrement recouverte par de minces muscles peauciers et leurs prolongements aponévrotiques qui s'étendent eux-mêmes sur les muscles sousjacents sous forme de fascia parfois très adhérents et extrêmement minces. Tout cet ensemble superficiel doit d'abord être soigneusement enlevé. On peut commencer à le détacher en partant de l'épine dorsale, où les expansions fibreuses ont été sectionnées par la séparation en deux demi-carcasses.

On se trouve alors en présence des premiers plans musculaires décrits, plus ou moins recouverts, selon l'état d'engraissement de l'animal, par une couche de gras externe qui comble également les dépressions ou intervalles entre les muscles. Avant d'aborder la dissection musculaire proprement dite, il convient encore d'enlever le plus complètement possible cette couche de graisse, et en particulier de bien en nettoyer les régions où les muscles se juxtaposent, afin d'éviter par la suite des hésitations ou des erreurs de dissection.

De même, au fur et à mesure du prélèvement musculaire, il est important d'éliminer le plus complètement possible, avant d'attaquer une nouvelle région, les dépôts de gras intermusculaires ainsi que tous éléments de tissu conjonctif, vaisseaux, ganglions ou nerfs de façon à bien assurer la netteté des plans musculaires à séparer. Toutes nos planches sont présentées après une telle préparation, ne laissant pratiquement en place que les muscles et les pièces osseuses.

\section{NOMENCLATURE, MORPHOLOGIE ET INSERTIONS MUSCULAIRES}

Le texte qui suit visant avant tout à permettre une identification correcte comportera essentiellement, sans autre considération, la nomenclature, la morphologie 
sommaire et les insertions de chaque muscle étudié. Éventuellement, quelques indications complémentaires seront données, pour une meilleure compréhension des schémas ou pour faciliter certaines étapes de la dissection.

La nomenclature utilisée comportera le nom français le plus courant ou le plus récent de chaque muscle, suivi de sa dénomination latine officielle de la nomenclature internationale Nomina Anatomica Veterinaria (HANOVRE, I963). Cette dernière nomenclature $(N . A . V$.) n'a été que tout récemment achevée, et demeure encore en voie de perfectionnement ( $R$. BARONE, I967).

Certains muscles de la région supérieure du cou, reliés à la tête, ont été évidemment sectionnés lors de la séparation de celle-ci. Nous en indiquerons cependant les insertions crâniennes. De même nous indiquerons également les insertions inférieures des muscles de la jambe ou de l'avant-bras, dont les tendons ont été sectionnés lors de la séparation de l'extrémité inférieure des membres.

Toutefois, comme il ne s'agit nullement d'un travail fondamental d'anatomie, les muscles seront décrits successivement selon leur ordre approximatif de prélèvement, correspondant à un déroulement plus aisé de la dissection, sans considération particulière soit de leur fonction, soit des régions anatomiques auxquelles ils appartiennent et pour lesquelles nous renvoyons aux ouvrages d'Anatomie vétérinaire.

Par ailleurs, pour plus de clarté des schémas, la demi-carcasse droite a été séparée en deux moitiés par une coupe passant entre la $9^{\mathbf{e}}$ et la Io $^{\mathbf{e}}$ vertèbres dorsales, laissant donc les 9 premières côtes sur la moitié antérieure, les 4 dernières côtes sur la moitié postérieure. (Signalons à ce sujet qu'il arrive assez fréquemment de trouver une côte surnuméraire, entière ou incomplète, et placée soit en avant de la première côte normale, soit en arrière de la dernière côte normale.)

Afin d'assurer une meilleure correspondance entre le texte et les schémas, nous distinguerons donc, d'une façon toute arbitraire, successivement 3 régions :

I - région antérieure : Cou et Tronc (pl. I à 6);

II - région postérieure : Tronc et Membre postérieur (pl. 7 à I5);

III - membre antérieur (p1. I6 à 20).

\section{I. - RÉGION ANTÉRIEURE : COU ET TRONC (pl. I à 6)}

\section{Trapèze (Trapezius) (pl. I).}

Large muscle plat, de forme approximativement triangulaire, s'étendant à la

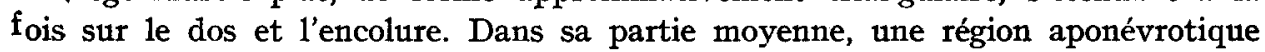
l'attache à la tubérosité de l'épine scapulaire. La partie supérieure est attachée par une mince aponévrose au long du ligament cervical et à la partie supérieure des apophyses épineuses des premières vertèbres thoraciques. La portion cervicale recouvre notamment la plus grande partie du rhomboïde.

\section{Brachio-céphalique (Brachiocephalicus) (pl. I-2).}

Muscle très allongé s'étendant sur le côté de l'encolure, de l'extrémité inférieure du bras au sommet de la tête, en recouvrant dans sa partie moyenne l'omo-tranversaire qu'il croise en y adhérant fortement ( $\mathrm{pl}$. I).

Il recouvre également, en cette région, la partie inférieure très mince de l'omohyoïdien (p1. 2). Il faut donc veiller, lors du prélèvement du brachio-céphalique 
(qu'on peut détacher d'abord à son extrémité inférieure) à bien le séparer de ces dewx derniers muscles.

La région supérieure se divise au niveau du tiers supérieur en deux branches faiblement distinctes :

- la branche inférieure assez étroite se prolonge par un tendon qui va s'attacher sur l'apophyse basilaire de l'occipital, en commun avec celui du sterno-céphalique et du muscle long de la tête,

- la branche supérieure fortement élargie se continue par un mince fascia qui rejoint d'une part celui du trapèze et d'autre part s'étale sur la nuque en se terminant au niveau de la corde du ligament cervical et de la ligne courbe de l'occipital.

La région inférieure s'insinue profondément entre le biceps et le brachial pour s'insérer en bas de la crête humérale.

\section{Omo-transversaire (Omotransversarius) (pl.I-2-3).}

Long ruban musculaire en partie recouvert par le brachio-céphalique auquel il adhère, sur la face latérale de l'encolure. L'extrémité supérieure est liée au splénius par un court tendon grâce auquel ces muscles se fixent sur l'aile de l'atlas. L'extrémité inférieure se termine en pointe pour aller s'attacher sur l'humérus dans la même région que le brachio-céphalique.

\section{Sterno-thyroïdien (Sternothyroideus) (p1. 2-3).}

Ce muscle et son homologue du côté opposé forment deux longues et grêles bandelettes recouvrant partiellement la trachée et accolées dans leurs tiers inférieur pour s'attacher selon une même insertion à l'extrémité antérieure du sternum, dans l'angle du $\mathrm{V}$ formé par les sterno-céphaliques. Un très court tendon situé en leur milieu les rend irrégulièrement digastriques, et chacune de ces bandelettes va se terminer dans la région supérieure sur un plan latéral du cartilage thyroïde.

\section{Sterno-hyoïdien (Sternohyoïleus) (p1. 2).}

Ce muscle et son homologue du côté opposé forment en avant de la trachée deux très minces bandelettes se détachant des muscles sterno-thyroïdiens au niveau du tendon qui les rend digastriques. Ces bandelettes croisent obliquement ces muscles pour aller s'attacher, dans la région supérieure, sur la partie médiane du corps de l'hyoïde, dans l'angle formé par les deux omo-hyoïdiens.

\section{Sterno-céphalique (Sternocéphalicus) (pl. I-2).}

Fort ruban musculaire situé le long de la face antérieure de l'encolure, prenant origine à sa partie inférieure sur le prolongement trachélien du sternum, et formant à cet endroit la pointe d'un $V$ avec son homologue du côté opposé. A la partie supérieure, les fibres musculaires se terminent au niveau de la base de l'aile de l'atlas et se continuent par une série de prolongements tendineux dont le principal va s'insérer sur l'apophyse basilaire de l'occipital, en commun avec celui du muscle long de la tête, ainsi qu'avec un prolongement tendineux du brachio-céphalique.

\section{Omo-hyoïdien (Omohyoideus (pl. 2).}

Minces bandelettes charnues remontant dans la région antéro-supérieure de l'encolure, les deux omo-hyoïdiens se réunissent à leurs extrémités supérieures, figu- 
rant la pointe d'un $\mathrm{V}$ renversé fixée sur la partie médiane du corps de l'hyoïde. Chacun de ces muscles croise obliquement le sterno-céphalique pour aller, sans atteindre l'épaule, se perdre sous le brachio-céphalique en une très mince cloison musculaire recouvrant partiellement le muscle long de la tête et les faisceaux supérieurs du scalène.

\section{Pectoral descendant (Pectoralis descendens) (pl. I-2).}

Muscle superficiel de forme rectangulaire, assez épais et foncé, antérieurement situé et attaché d'une part sur la région antérieure du sternum, d'autre part sur la crête humérale et sur la gaine aponévrotique du biceps, en commun avec la portion antérieure du pectoral transverse.

\section{Pectoral transverse (Pectoralis transversus) (pl. I-2-3).}

Large muscle quadrilatère, assez pâle et mince, comportant deux portions distinctes :

- une portion antérieure, un peu plus épaisse , croise en $\mathrm{X}$ le pectoral descendant qui la recouvre partiellement et qui présente les mêmes insertions,

- une portion postérieure, plus large mais mince et pâle, s'attache d'une part sur la région moyenne du sternum, d'autre part descend sur la face interne de l'avantbras où elle rejoint le fascia superficiel.

\section{Io. Pectoral ascendant (Pectoralis ascendens) (pl. I-2.-3).}

Vaste muscle recouvrant la région latérale antérieure de la cage thoracique et s'insinuant sous le bras jusqu'au niveau de l'épaule.

- Attaches inférieures : le long de la ligne sternale, au niveau des trois dernières sternèbres, et plus postérieurement sur la tunique abdominale.

- Attaches supérieures: au niveau de l'apophyse coracoïde, sur l'aponévrose d'enveloppe de la partie supérieure du tendon du biceps ; sur l'humérus au niveau du trochin et de la coulisse bicipitale; enfin sur l'aponévrose d'enveloppe du coracobrachial.

\section{Pectoral scapulaire (Pectoralis cleidoscapularis) (p1 . 2-3).}

Très fine bandelette musculaire noyée dans l'amas graisseux pré-scapulaire, attachée d'une part à l'extrémité antéro-latérale du sternum à la hauteur de 1'insertion du sterno-céphalique et tangentiellement à celui-ci, se terminant d'autre part à la face interne du brachio-céphalique au niveau du tiers inférieur.

12. Grand dorsal (Latissimus dorsi) (p1. I et aussi pl. 7).

Large muscle dont les fibres sont étalées en éventail vers l'arrière, sur la face latérale et supérieure de la cage thoracique, et s'insinuant vers l'avant en un fuseau effilè sous les muscles du bras. Il se continue vers l'arrière par un très large fascia aponévrotique étalé sur les régions postérieures, et qui contribue notamment à la formation du fascia dorso-lombaire.

Le fuseau antérieur se continue par un tendon aplati qui vient contourner à sa base le tendon du muscle Grand-rond pour venir s'insérer en commun avec celui-ci au niveau du tiers supérieur de la face interne de l'humérus. 
Après enlèvement de ce muscle et à ce stade de la dissection, l'épaule 'n'est plus rattachée au tronc que par le Rhomboïde et le Grand dentelé, insérés notamment dans la région supérieure du scapulum.

\section{Rhomboïde (Rhomboïdeus) (p1. 3).}

Situé sous la portion cervicale du Trapèze, c'est un muscle plat de forme quadrangulaire, prolongé vers l'avant en pointe effilée fixée sur la corde du ligament cervical. Au niveau du garrot il s'attache au sommet des apophyses épineuses des premières vertèbres thoraciques et dans sa région inférieure il s'insère sous le cartilage de prolongement du scapulum et à l'extrémité antéro-supérieure de cet os.

14. Grands dentelés (Serratus ventralis cervicis et serratus ventralis thoracis) (pl. I-3).

Chez le mouton, dentelé du cou et dentelé du thorax ne sont pas distincts (comme chez le bœuf) et se présentent, bien que conservant leurs deux noms, comme un seul énorme muscle auquel on peut donner l'appellation commune de Grand dentelé (selon Bourgelat, cité pat R. TAGaND et R. BARONE).

C'est un puissant muscle disposé en éventail contre la paroi thoracique, recouvert partiellement par l'épaule qu'il déborde en avant et en arrière et dont l'extrémité inférieure présente une série de dentelures. Dans la région thoracique, ces dentelures s'attachent sur la face externe des 9 premières côtes, et dans la région cervicale sur les apophyses transverses des 5 dernières vertèbres cervicales. Dans la région supérieure, les fibres convergent vers l'extrémité supérieure du scapulum sous lequel elles s'insèrent, de part et d'autre du muscle sous-scapulaire, par une large aponévrose fixée sur les surfaces d'insertion des grands dentelés, ainsi que sur le bord postéroinférieur du cartilage de prolongement $d u$ scapulum. A ce stade de la dissection l'épaule est alors complètement séparée du tronc et peut être enlevée avec les régions inférieures du membre antérieur pour une dissection ultérieure.

\section{I5. Splenius (Splenius) (p1. I-3).}

Muscle mince, étalé sur la face latérale et supérieure de l'encolure. Sa région antéro-supérieure se rattache au ligament cervical et vers l'avant à la crête mastoïdienne par une portion aponévrotique qui rejoint celle du petit complexus. Sa région antéro-inférieure rejoint l'attache de l'omo-transversaire au niveau de 1'aile de 1'atlas. La région postérieure aponévrotique se termine au sommet des apophyses épineuses des premières vertèbres dorsales.

\section{I6. Cervical ascendant (Iliocostalis cervicis) (pl. 4).}

Petit muscle fusiforme situé entre le scalène et le transversaire du cou, dans le prolongement du long costal. Il est attaché à son extrémité inférieure vers le sommet de la première côte, et inséré d'autre part surles apophyses transverses des $5^{\mathrm{e}}, 6^{\mathrm{e}}$ et $7^{\mathbf{e}}$ vertèbres cervicales.

\section{I7. Scalène (Scalenus) (pl. 3-4).}

Muscle de la base de 1'encolure, divisé supérieurement en 5 faisceaux parallèles attachés respectivement aux apophyses transverses des 5 dernières vertèbres cervicales. Les 4 faisceaux inférieurs se rejoignent à leur base pour s'attacher en commun tout au long de la partie moyenne antérieure de la première côte. Le faisceau supé- 
rieur, plus court, est légèrement séparé du groupe précédent par une fente d'où émergent les nerfs du plexus brachial. Il relie l'extrémité proximale de la première côte à l'apophyse transverse de la $7^{\mathfrak{e}}$ vertèbre cervicale.

\section{I8. Droit du thorax (Rectus thoracis) (pl. 4).}

Petit muscle fusiforme qui croise obliquement les premiers cartilages sternocostaux. Son extrémité supérieure s'attache à la base de la face externe de la première côte. L'extrémité inférieure est attachée par un tendon fibreux sur les cartilages de prolongement des $2^{\mathrm{e}}, 3^{\mathrm{e}}$ et $4^{\mathrm{e}}$ côtes.

\section{I9. Long de la tête (Longus capitis) (pl. 3-4).}

Muscle très allongé de la base de l'encolure, accompagnant partiellement le long du cou, et qri s'étend de l'apophyse transverse proéminente de la $6^{\mathrm{e}}$ vertèbre cervicale jusqu'au corps du sphénoïde et à l'apophyse basilaire de l'occipital où se fait son insertion supérieure, très proche de celle de son homologue du côté opposé. Sur son trajet, il lance des digitations qui vont s'attacher sur les apophyses transverses des $3^{\mathbf{e}}$, $4^{\mathrm{e}}$ et $5^{\mathrm{e}}$ vertèbres cervicales.

Chez le Mouton, comme chez les Bovins, ce muscle est doublé par un fort faisceau musculeux (trachélo-atloïdien, de CHAUVEAU).

20. Trachélo-atloïdien (Muscle non mentionné dans les N.A.V.) (pl. 3-4).

Il possède avec le précédent une attache inférieure commune sur l'apophyse transverse de la $6^{\mathbf{e}}$ vertèbre cervicale, et il s'attache d'autre part sur les apophyses transverses des $3^{\mathrm{e}}, 4^{\mathrm{e}}$ et $5^{\mathrm{e}}$ vertèbres cervicales, pour se terminer dans sa partie supérieure par une insertion à la base de l'aile de l'atlas.

\section{I. Petit complexus (Mm. longissimus capitis et longissimus atlantis) (p1. 4).}

Muscle étroit et allongé, étendu sous le splénius sur la face latérale de l'encolure semblant continuer dans sa région antérieure les faisceaux du transversaire du cou, et recouvrant partiellement le grand complexus.

A sa partie supérieure il se divise en deux faisceaux distincts dont les prolongements tendineux vont s'attacher, pour le faisceau supérieur sur l'apophyse mastoïde du temporal, pour le faisceau inférieur à la base de l'aile de l'atlas.

Les languettes de la région inférieure s'attachent sur les apophyses articulaires des 6 dernières vertèbres cervicales et, en commun avec celles du grand complexus, sur les apophyses transverses des 2 premières vertèbres dorsales.

\section{Grand complexus (Semispinalis capitis) (p1. 4).}

Un des plus forts muscles de l'encolure, en forme de long triangle incurvé, plaqué dans sa moitié supérieure contre la portion lamellaire du ligament cervical.

Son extrémité supérieure prolongée par un fort et large tendon aponévrotique se fixe par ce dernier sur la protubérance occipitale ainsi que sur le côté du ligament cervical.

Les languettes de sa région ventrale se fixent par de courts tendons sur les apophyses articulaires des 6 dernières vertèbres cervicales, puis sur les apophyses transverses des $6 \cdot$ (ou 7 ) premières vertèbres dorsales. 
L'extrémité postéro-inférieure du muscle (correspondant à ces dernières languettes fixées sur les premières vertèbres dorsales) s'engage entre le long dorsal et le long épineux du dos qu'il est nécessaire d'écarter l'un de l'autre à ce niveau pour pouvoir atteindre cette extrémité du grand complexus.

\section{Transversaire du cou (Longissimus cervicis) (p1. 4-5).}

Muscle constitué par une série de faisceaux successifs couchés à la base des complexus, semblant continuer le long dorsal dans la région de l'encolure, et se terminant en arrière en une pointe effilée qui atteint le niveau de la tête de la $7^{\ominus}$ côte. Dans sa région dorsale, il se fixe sur les apophyses transverses des 6 premières vertèbres dorsales. Dans sa portion cervicale, les faisceaux se terminent par une région aponévrotique se fixant respectivement sur les apophyses transverses des $3^{\mathrm{e}}$ à $7^{\mathbf{e}}$ vertèbres cervicales.

De même que pour le muscle précédent, pour atteindre les faisceaux de 1'extrémité postéro-inférieure, il faut écarter l'une de l'autre les extrémités antérieures des muscles long dorsal et long épineux du dos.

\section{Epineux du cou (Spinalis cervicis) (p1. 5).}

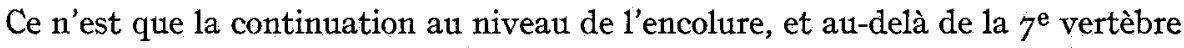
cervicale, du long épineux du dos ( $\left.\mathbf{n}^{\circ} 37\right)$ dont il n'est pratiquement pas séparable et qui se termine au niveau de la $3^{\mathbf{e}}$ vertèbre cervicale, entre le grand complexus et la lame du ligament cervical.

\section{Multifide du cou (Multifidus cervicis) (p1. 5).}

Ce muscle est formé de 5 faisceaux successifs dirigés obliquement de bas en haut et d'arrière en avant, et qui continuent dans la région de l'encolure ceux du multifide dorso-lombaire. Ils sont attachés à la base sur les tubercules articulaires des 5 dernières vertèbres cervicales, et à leur extrémité supérieure sur les apophyses épineuses des $2^{\mathbf{e}}$ à $6^{\mathbf{e}}$ vertèbres cervicales.

\section{Intertransversaire du cou (Intertransversarii cervicis) (p1. 5).}

Correspondant aux 6 dernières vertèbres cervicales, ce sont de petits faisceaux musculaires logés dans la gouttière latérale comprise entre les apophyses transverses et les apophyses articulaires, et dont les uns joignent entre elles les apophyses transverses successives, les autres reliant obliquement une apophyse transverse à 1'apophyse articulaire de la vertèbre suivante.

\section{Long du cou (Longus colli) (p1. 5-6).}

Muscle très allongé et puissant, s'étendant tout au long de la face ventrale des vertèbres cervicales et des 6 premières vertèbres thoraciques. Sur la ligne médiane, il est étroitement accolé à son homologue du côté opposé, au point que les anatomistes vétérinaires ont souvent décrit l'ensemble comme un seul muscle impair. Pour chaque long du cou, deux portions peuvent être distinguées : une portion thoracique et une portion cervicale.

- La portion thoracique s'insère sur les corps vertébraux des 6 premières vertèbres thoraciques, et s'attache vers l'avant par un fort tendon sur la cuspide postérieure de l'apophyse transverse proéminente de la $6^{\mathbf{e}}$ vertèbre cervicale. 
- La portion cervicale lance une série de faisceaux obliques et étroitement imbriqués qui s'attachent successivement : en dehors aux apophyses transverses des 6 dernières vertèbres cervicales; en dedans sur la crête inférieure et la face latérale du corps des 6 premières vertèbres cervicales. Le faisceau antérieur se termine à l'avant par un court tendon fixé sur le tubercule inférieur de l'atlas.

(La planche 6 montre le long du cou dans son ensemble, tel qu'il peut être vu du côté de la face interne de la carcasse.)

\section{Grand oblique de la tête (Obliquus capitis caudalis) (p1. 5).}

Court et large muscle inséré tout au long du bord de l'aile de l'atlas, et d'autre part sur le côté de l'apophyse épineuse de l'axis.

\section{Grand droit dorsal de la tête (Rectus capitis dorsalis major) (p1. 5).}

Muscle plat étalé au niveau de la nuque, sous l'aponévrose supérieure du grand complexus avec laquelle il s'insère sur la protubérance occipitale. Son extrémité inférieure s'attache le long de l'apophyse épineuse de l'axis.

Il est doublé en dessous par un petit muscle complémentaire qui est le petit droit dorsal de la tête.

30. Petit droit dorsal de la tête (Rectus capitis dorsalis minor) (non représenté sur la planche 5 , il apparaît après enlèvement du grand droit dorsal de la tête).

C'est un petit muscle aplati qui s'étale à la partie postérieure de la capsule occipito-atloïdienne. Il est inséré sur la protubérance occipitale près du ligament cervical, et d'autre part sur la face postérieure du corps de 1'atlas.

\section{3r. Petit oblique de la tête (Obliquus capitis cranialis) (p1. 5).}

Muscle d'aspect prismatique inséré tout au long du bord de l'aile de 1'atlas. I1 est attaché d'autre part dans la région latéro-supérieure de la face nuchale de l'occipital, et sur l'apophyse mastoìde du temporal.

\section{Droit ventral de la tête (Rectus capitis ventralis) (non représenté).}

Petit muscle rectangulaire allongé recouvert par le tiers supérieur du muscle long de la tête. Il possède les mêmes insertions supérieures que ce dernier, et se fixe à sa partie inférieure sur le bord postérieur de la face ventrale de l'atlas.

\section{Droit latéral de la tête (Rectus capitis lateralis) (non représenté).}

Il correspond à une petite bandelette musculaire située entre les deux muscles précédents. Il joint l'arc ventral de l'atlas à la face interne de l'apophyse styloỉde de l'occipital.

A ce stade de la dissection il reste encore, sur la région antérieure décrite de la demi-carcasse, les portions antérieures des muscles dorso-lombaires ainsi que celles des muscles abdominaux.

Ces différents muscles seront décrits avec ceux de la région postérieure. Les muscles intercostaux, sus-costaux et transverse du thorax ne seront pas décrits. 


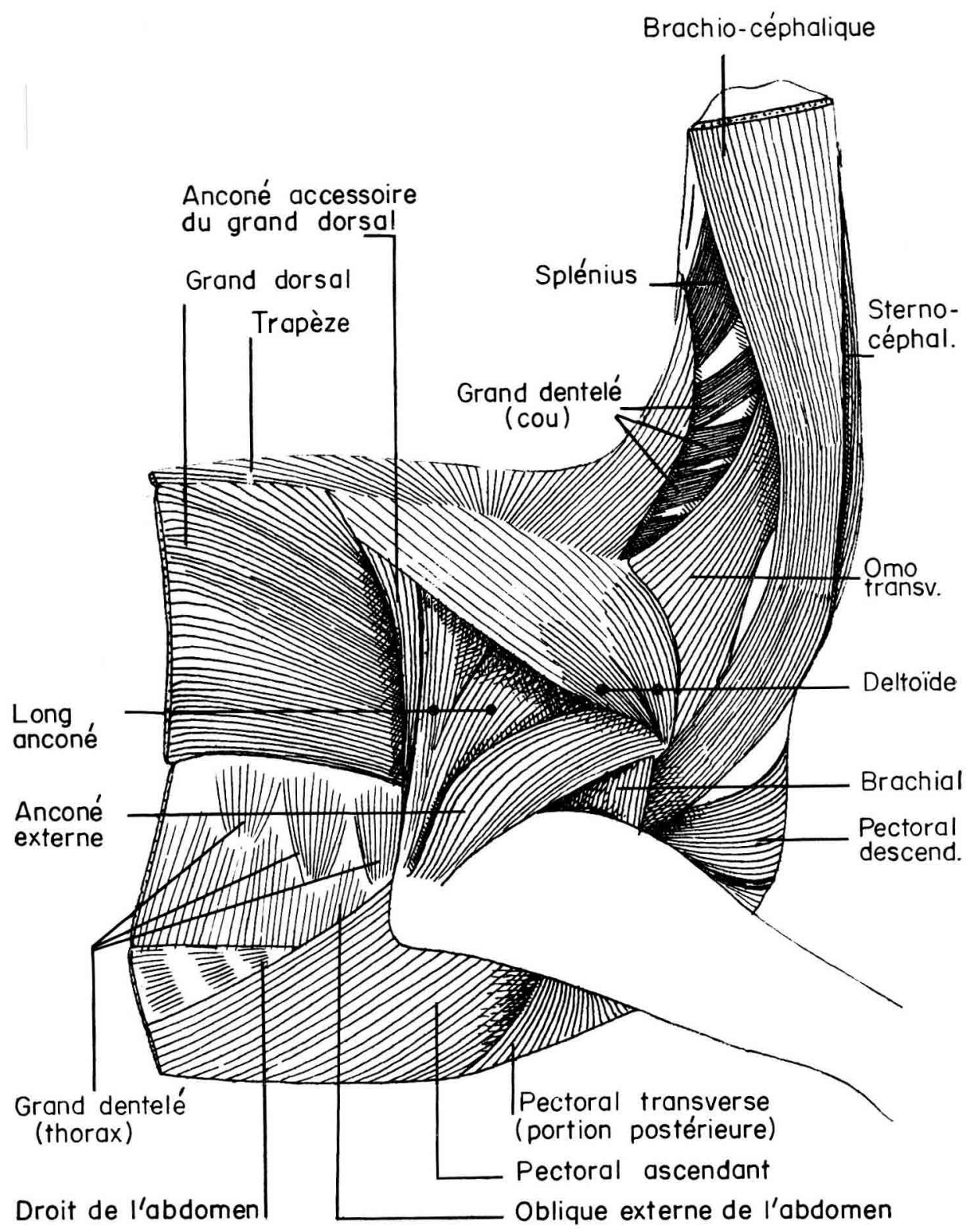

M.Bet D.F 


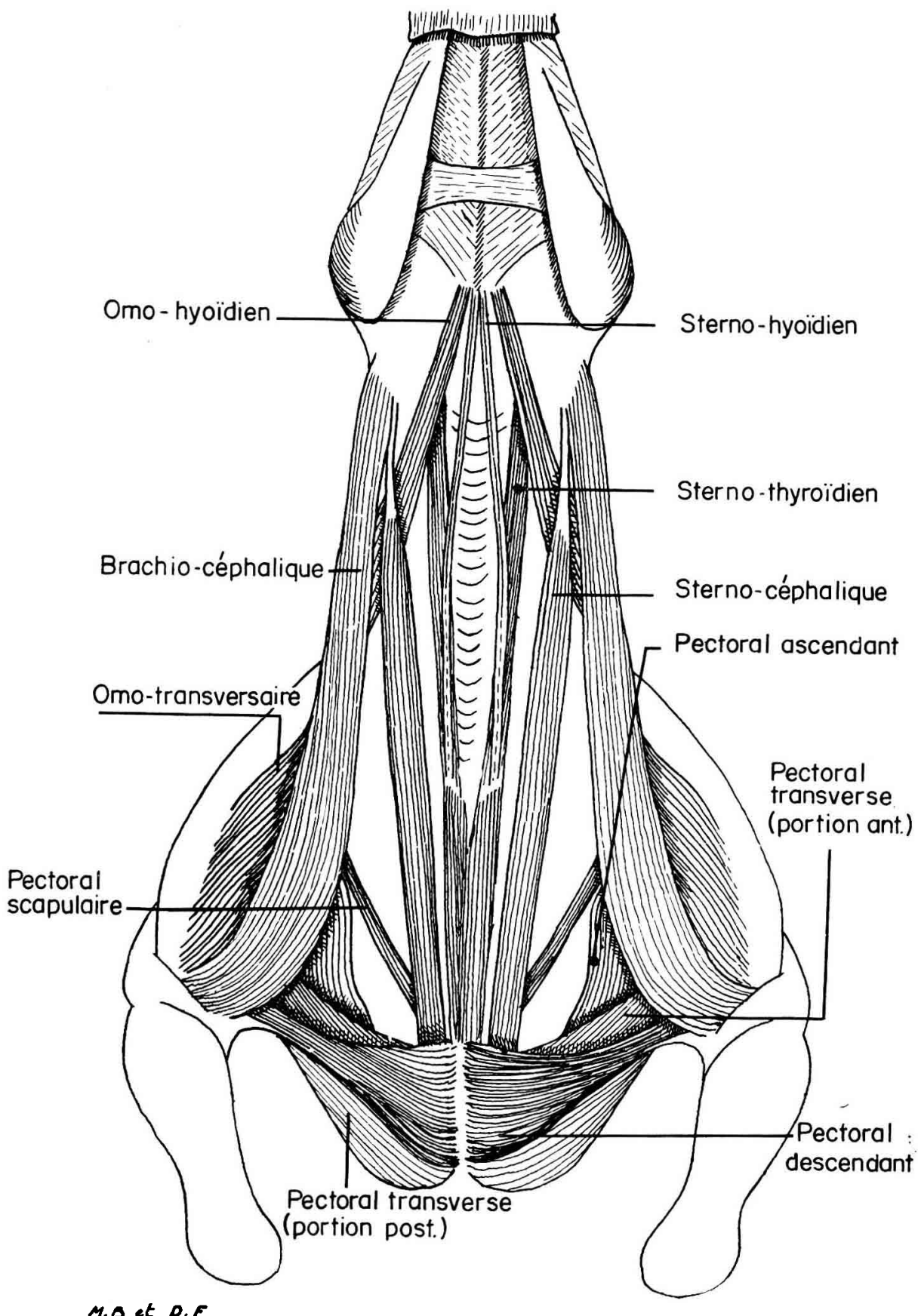

Planche 2. - Vue de face 


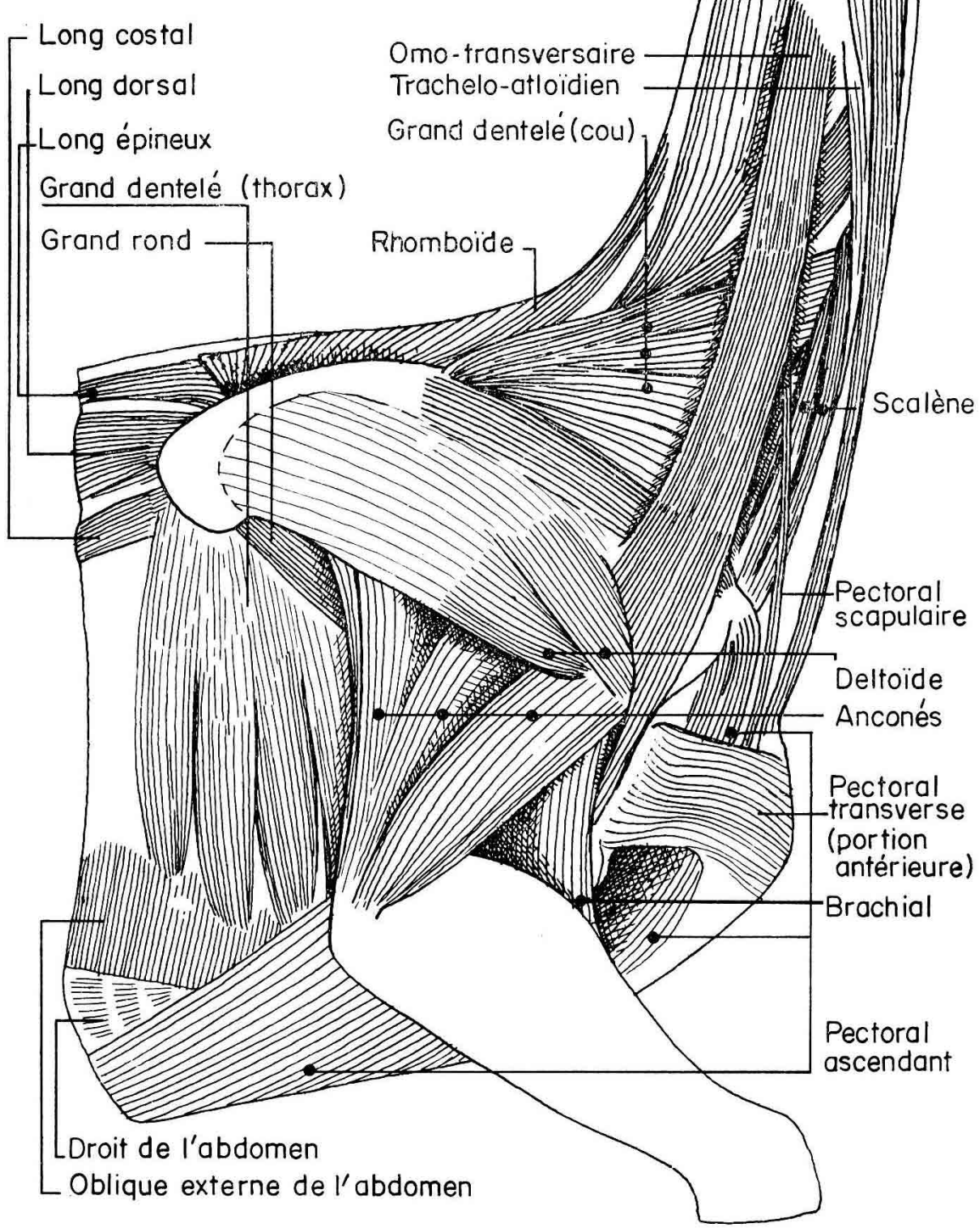

Sterno - thyroidien

Long de la tête

Splénius
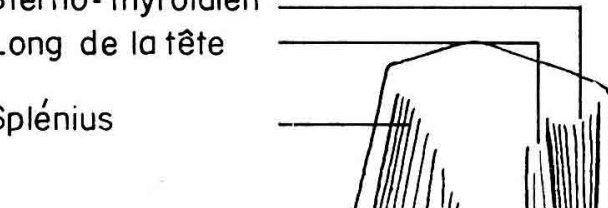


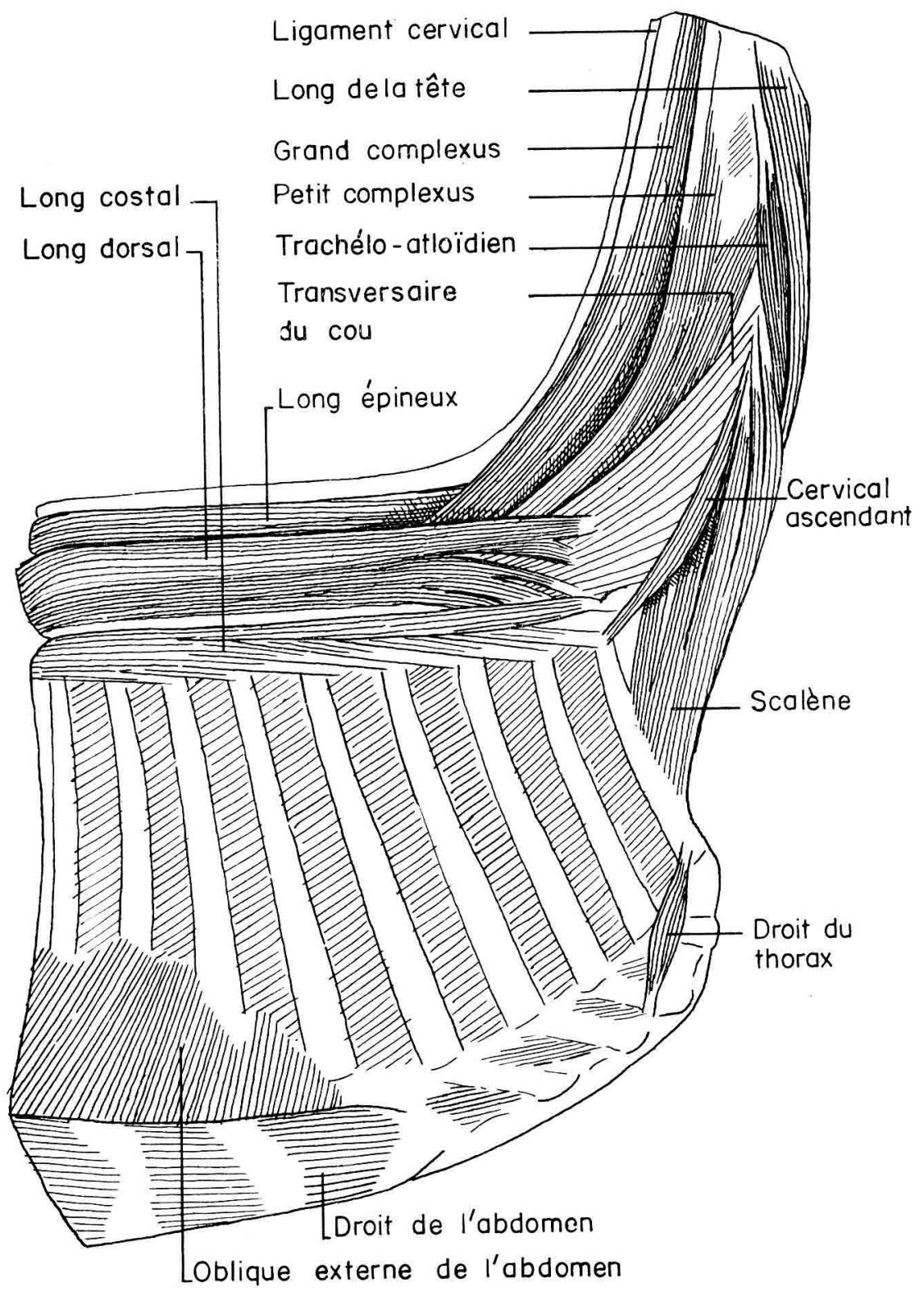

M. B et o.F 


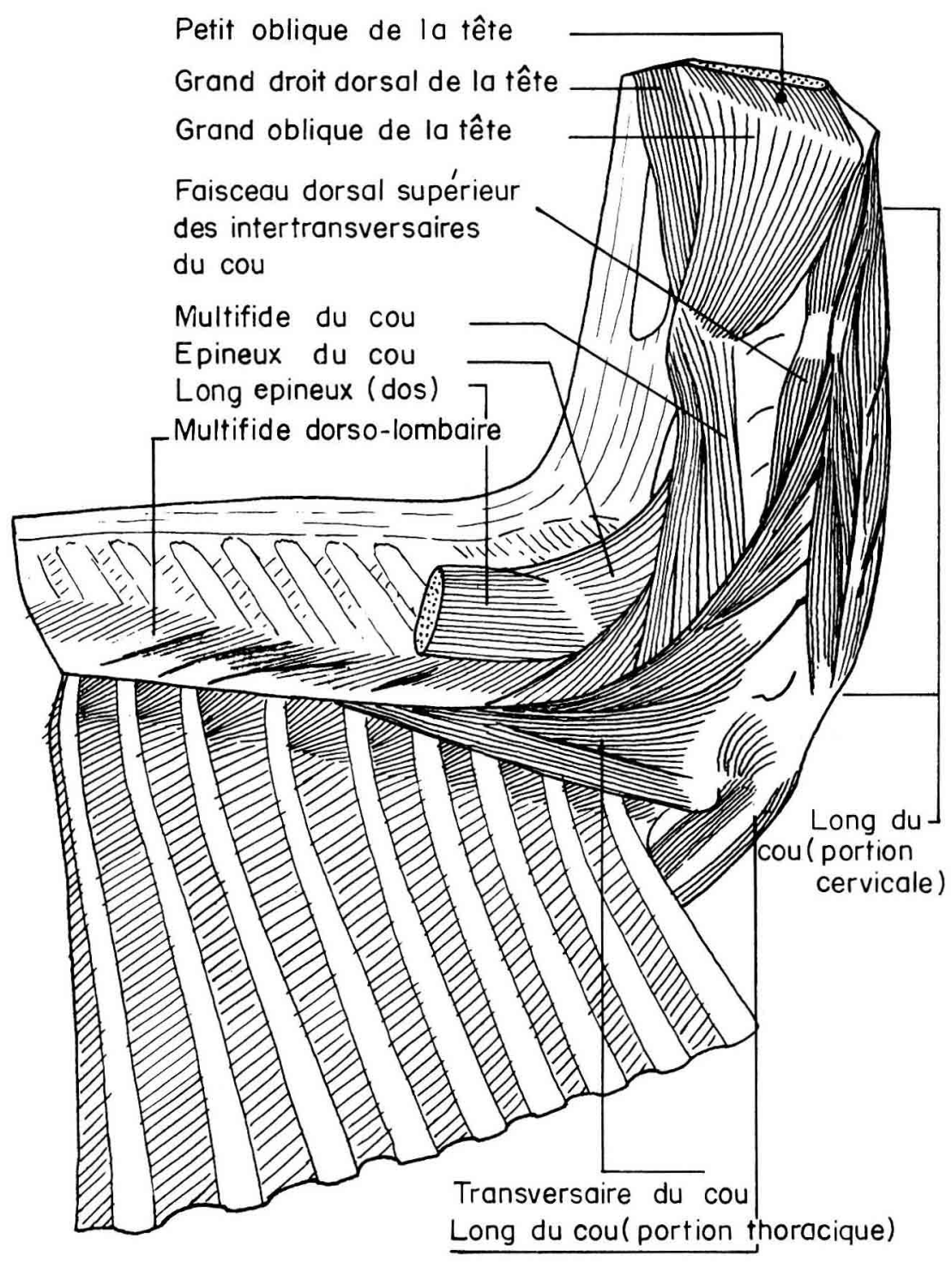

M.B et D.F 


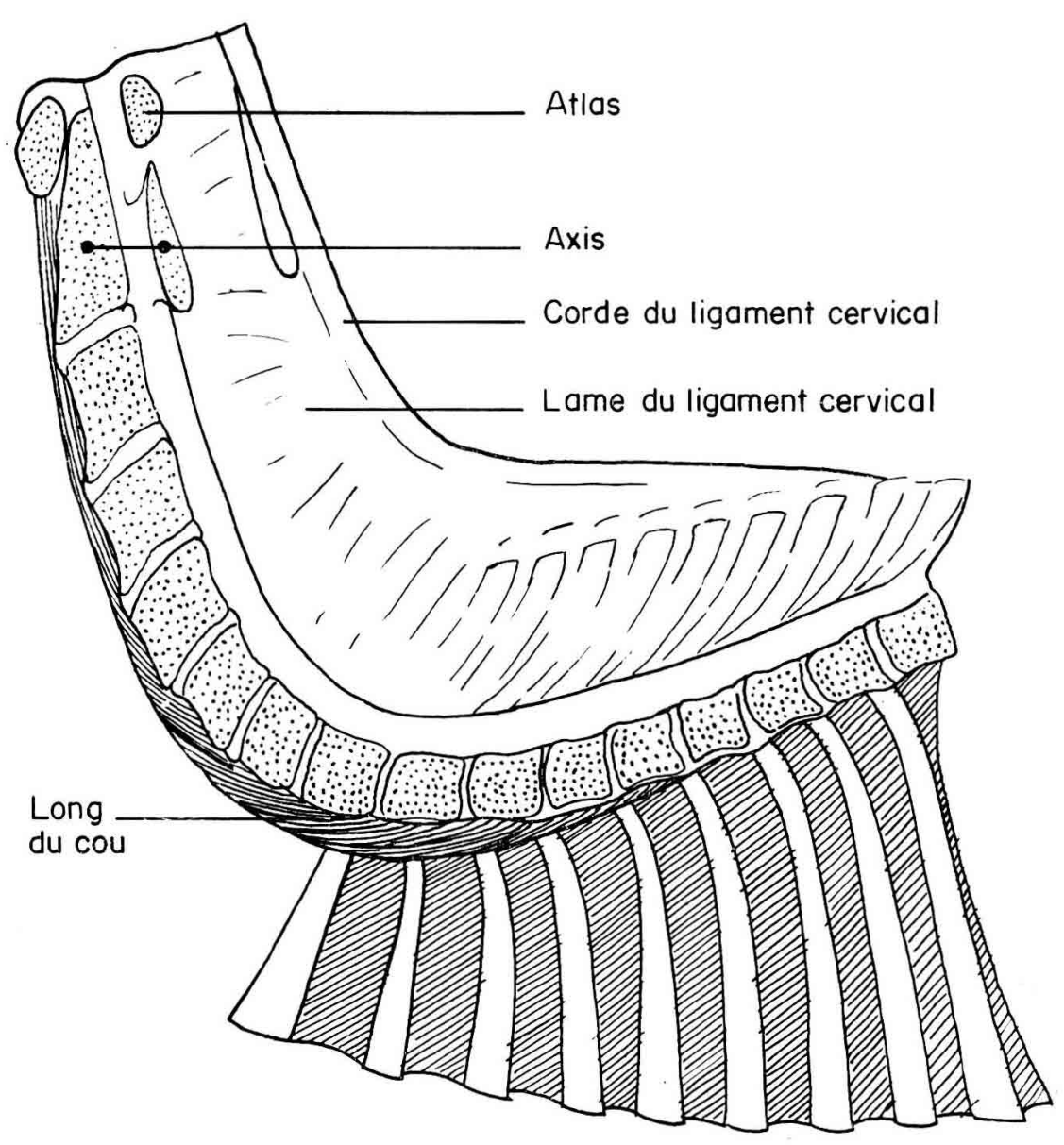

MB et $0 . F$

Planche 6. - Vue interne 


\section{II. - RÉGION POSTÉRIEURE : TRONC ET MEMBRE POSTÉRIEUR (p1. 7 à I5)}

\section{Petit dentelé caudal (Serratus dorsalis caudalis) (p1. 7).}

Petit muscle de la région postéro-supérieure du thorax, qui présente une série de 5 dentelures correspondant aux 4 derniers espaces intercostaux et au muscle rétracteur de la dernière côte. Chacune des ces dentelures est attachée le long du bord postérieur proximal de la côte correspondante. La partie supérieure commune se prolonge par un fascia aponévrotique qui va se confondre avec le fascia dorsolombaire.

\section{Long costal (Iliocostalis thoracis) (p1. 8 et aussi 3 et 4 ).}

Muscle rubanné de la région supra-costale, s'allongeant de la base de l'encolure jusqu'aux vertèbres lombaires. Il est constitué par une série de faisceaux imbriqués dont les tendons, obliques vers l'avant et de haut en bas, et parallèles entre eux, sont fixès à la partie supérieure des côtes successives et chevauchent les deux ou trois côtes suivantes. Le faisceau le plus antérieur s'attache sur l'apophyse transverse de la dernière vertèbre cervicale. Le dernier faisceau, terminé en pointe, après avoir chevauché la partie supérieure des trois dernières côtes, va s'attacher par une expansion fibreuse sur les apophyses transverses des premières vertèbres lombaires.

\section{Long dorsal (Longissimus thoracis) (p1. 7 et aussi pl. 3.4).}

- Le plus volumineux des muscles du corps, et formant la masse musculaire la plus importante de la région dorso-lombaire, il s'étend au long de la gouttière vertébro-costale depuis le sacrum jusqu'à la base de l'encolure.

- Diminuant d'épaisseur de l'arrière vers l'avant, il est revêtu d'une aponévrose d'enveloppe nacrée qui diminue d'importance de l'arrière à l'avant, pour s'effacer complètement à l'extrémité antérieure. Il est d'autre part recouvert par les expansions fibreuses du fascia dorso-lombaire. Enfin son extrémité postéro-supérieure est recouverte par une petite expansion du muscle fessier moyen.

- Postérieurement et dans sa région supérieure, il se termine en pointe dans l'angle sacro-iliaque par un fort tendon attaché aux apophyses épineuses sacrées. Le reste de la région postérieure s'attache sur le bord antérieur de l'ilium ainsi qu'à la face interne de celui-ci. Cette région mêle quelques fibres avec celles des premiers faisceaux des muscles sacro-coccygiens. Tout au long de la gouttière vertébro-costale, le long dorsal est attaché sur les apophyses mamillaires des vertèbres lombaires et thoraciques, sur les apophyses transverses des vertèbres lombaires et sur la face externe de 1'extrêmité supérieure des côtes. Il se termine antérieurement (P1.4) par 4 languettes minces recouvrant partiellement le transversaire du cou, et qui s'attachent respectivement sur les apophyses transverses des $5^{\mathrm{eme}}, 6^{\mathrm{eme}}, 7^{\mathrm{eme}}$ vertèbres cervicales et de la I ere vertèbre thoracique.

- Au niveau de la région lombaire, les fibres tendineuses du muscle long épineux, plus ou moins mêlées à celles du multifide dorso-lombaire, viennent également s'attacher à celles du long dorsal. 
37. Long épineux (Spinalis thoracis) (pl. 7-8 et aussi p1. 3-4-5).

Muscle de la région dorso-lombaire, formé par une série de faisceaux parallèles, dont la masse charnue antérieure ne dépasse pas vers l'arrière la région thoracique. Cette masse charnue, plaquée contre le ligament cervical, couvre la région supérieure de la partie thoracique du long dorsal (pl. 4). Elle se prolonge postérieurement par une série de faisceaux tendineux parallèles qui vont s'attacher sur les apophyses épineuses et sur les apophyses mamillaires des premières vertèbres lombaires et des dernières thoraciques. Ces faisceaux serrés entre eux, forment une large bande aponévrotique dont certaines fibres vont se mêler à celles du ligament cervical, d'autres aux fibres tendineuses du multifide dorso-lombaire, et d'autres à celles du long dorsal. (Sur la planche 8 , où le muscle long dorsal a été enlevé cette région postérieure du long épineux a été relevée et épinglée, a fin de mieux découvrir le multifide dorso-lombaire.)

Antérieurement (p1.5) le muscle ne se distingue pas de l'épineux du cou (no 24) qui lui fait suite.

\section{Multifide dorso-lombaire (Multifidus) (pl. 8 et aussi pl. 5).}

Muscle le plus profond de la région dorso-lombo-sacrée, attaché postérieurement sur la lèvre latérale du sacrum. Il est formé d'une série de faisceaux obliques d'arrière en avant et de bas en haut, et attachés d'une part sur les tubercules mamillaires des vertèbres lombaires et les apophyses transverses des vertèbres thoraciques, d'autre part sur les apophyses épineuses des vertèbres immédiatement précédentes. I1 se continue antérieurement par le multifide du cou (pl. 5).

On trouve également dans la région lombaire et dorsale postérieure une série de courts faisceaux qui dessinent avec les précédents la deuxième branche d'un $\mathrm{V}$ ouvert à l'avant. Ces faisceaux unissent les tubercules mamillaires des vertèbres lombaires à la base de 1'apophyse transverse de la vertèbre précédente, et dans la région thoracique 1'apophyse articulaire antérieure à la base de l'apophyse transverse de la vertèbre précédente.

Nous ne les avons pas trouvés décrits dans les ouvrages classiques d'anatomie consultés. Ils nous semblent devoir correspondre aux faisceaux dorsaux identiquement disposés des intertransversaires du cou.

\section{Muscles abdominaux}

Les muscles abdominaux, qui correspondent aux parois de l'abdomen, sont disposés en couches successives et sont doublés extérieurement par une vaste expansion fibro-élastique : la tunique abdominale, qui contribue au soutien des viscères.

Les muscles abdominaux dechaque moitié du corps rejoignent leurs fibres ou expansions fibreuses sur la ligne ventrale médiane pour former une corde fibreuse dite ligne blanche étendue du sternum au pubis où elle s'étale pour contribuer à la formation du tendon prépubien inséré sous le pubis, un peu en arrière de son bord antérieur.

Du plan superficiel externe au plan interne profond on peut distinguer successivement les muscles suivants :

Oblique externe de l'abdomen ou grand oblique,

Oblique interne de l'abdomen ou petit oblique,

Droit de l'abdomen, Transverse de l'abdomen. 
39. Oblique externe de l'abdomen (Obliquus externus abdominis) (pl. 7 et aussi pl.r-3-4).

Très large muscle de la paroi latérale de l'abdomen et du thorax. Il présente deux portions : une portion charnue ; une portion aponévrotique.

- La portion charnue, dans sa partie antérieure, s'étale en se fixant à la base de toutes les côtes asternales et des dernières sternales. Ce faisant, elle dessine une série de festons charnus dont les premiers s'imbriquent avec les derniers festons correspondants du grand dentelé (p1. I-3). Par son bord supérieur, depuis la dernière côte jusqu'à l'angle externe de l'ilium, elle est insérée sur le fascia dorso-lombaire qui recouvre les muscles de cette région.

- La portion aponévrotique, qui représente toute la région postéro-inférieure, aussi étendue que la précédente à laquelle elle fait suite, est en rapport avec le muscle oblique interne et va se fixer sur la ligne blanche, sur le tendon prépubien et sur l'angle externe de 1'ilium.

L'ensemble du muscle est doublé extérieurement par la tunique abdominale.

\section{Oblique interne de l'abdomen (Obliquus internus abdominis) (p1. 8-9).}

C'est un large muscle dont les fibres croisent en $\mathrm{X}$ celles de l'oblique externe qui le recouvre entièrement. Comme ce dernier il présente une portion charnue et une portion aponévrotique.

- La portion charnue, plus épaisse dans sa région postérieure, où ses fibres rayonnent à partir de l'angle externe de l'ilium, s'amincit beaucoup dans sa région antérieure qui présente une étroite fenêtre à travers laquelle on distingue les fibres du muscle transverse sous-jacent (p1. 8). Elle est attachée postérieurement à l'arcade crurale et à l'angle externe de l'ilium. Son bord supérieur est relié au fascia dorsolombaire et au muscle rétracteur de la dernière côte.

- La portion aponévrotique fait suite à la portion charnue en s'étalant jusqu’à la ligne blanche après avoir auparavant mêlé ses fibres avec celles correspondantes de 1'aponévrose de l'oblique externe. Elle est insérée d'autre part sur le tendon prépubien ainsi qu'à la base de la dernière côte et sur les cartilages de prolongement asternaux des 3 ou 4 dernières côtes.

\section{I. Droit de l'abdomen (Rectus abdominis) (pl. 7-8-9 et aussi I-3-4).}

Forte bande musculaire allongée du sternum au pubis. Son bord interne, rectiligne, correspond à la ligne blanche. Son bord externe présente une courbure régulière demi-elliptique. Postérieurement il est inséré sur le tendon prépubien. Antérieurement il s'attache à l'extrémité postéro-inférieure du sternum, à la base de l'appendice xyphoïde, et par de courtes aponévroses sur les prolongements cartilagineux des 4 ou 5 dernières côtes sternales.

- Les fibres musculaires sont entrecoupées, dans leur trajet longitudinal, par une série de lignes fibreuses dessinant au long du muscle une succession de bandes blanchâtres parallèles, d'aspect caractéristique.

La face externe du muscle est recouverte par les aponévroses de l'oblique externe et de l'oblique interne, confondues à son niveau. La face interne est tapissée par la portion aponévrotique du transverse de l'abdomen. 
42. Transverse de l'abdomen (Transversus abnominis) (p1. 8-9).

Le plus profond des muscles abdominaux. Il présente comme les obliques une portion charnue large et plate suivie d'une vaste portion aponévrotique.

- La portion charnue s'insère à la face interne des cartilages de prolongement de toutes les côtes asternales et à la face interne de la base de la dernière côte, puis par un court fascia prolongeant le bord supérieur, à l'extrémité des apophyses transverses des vertèbres lombaires successives.

- La portion aponévrotique, qui tapisse en particulier la face interne du droit de l'abdomen, s'attache en avant sur l'appendice xyphoïde, puis ensuite tout au long de la ligne blanche.

43. Rétracteur de la dernière côte (pl. 8-9) (non désigné dans les $N . A . V$. ; avait été nommé par la Commission de 1897 Retractor ultimae costae).

Petite bande musculaire située sous la dernière languette postérieure du petit dentelé caudal. Elle s'étend du bord postérieur de la partie haute de la dernière côte aux apophyses transverses des 2 ou 3 premières vertèbres lombaires.

\section{Petit psoas (Psoas minor) (pl. 9).}

Muscle le plus interne de la région sous-lombaire, dont le corps charnu est constitué par une série de faisceaux délicats directement insérés à la face inférieure du corps des vertèbres lombaires, ainsi que de la dernière vertèbre thoracique. Ces faisceaux sont reliés à un tendon inférieur commun qui prolonge le corps charnu pour aller s'étaler sur le bord inférieur du col de l'ilium au niveau de la crête iléo-pectinée.

\section{Grand psoas (Psoas major) (p1. 9-Io).}

Muscle de la région sous-lombaire, de forme analogue à celle du petit psoas, contre lequel il est allongé. Ses fibres s'attachent inférieurement à la face inférieure du corps et sur la face ventrale des apophyses transverses des vertèbres lombaires, et vers l'avant elles se terminent sur la dernière vertèbre thoracique ainsi que sur la face interne supérieure de la dernière côte.

La région postérieure s'insinue en pointe entre les deux branches de l'iliaque (pl. ro) et s'insère par un tendon commun avec celui de ce dernier sur le trochantin du fémur.

\section{Carré des lombes (Quadratus lumborum) (p1. 9-1o).}

Muscle de la région sous-lombaire profonde, situé sous le grand psoas qui n'en laisse apparaître que les faisceaux les plus antérieurs.

Il prend origine au niveau du bord latéral interne de l'articulation sacro-iliaque, et s'allonge vers l'avant en une série de faisceaux pennés plus ou moins chevauchants, plaqués sur la face ventrale des apophyses transverses des vertèbres lombaires sur lesquelles il s'insère, et également pour les faisceaux les plus avancés sur la face inférieure du corps des $12^{\mathrm{e}}, \mathrm{II}^{\mathrm{e}}$ et $\mathrm{IO}^{\mathrm{e}}$ vertèbres thoraciques. Ces faisceaux antérieurs sont également attachés à la face interne supérieure des trois dernières côtes.

47. niaque (Iliacus) (pl. 8-Io-II-I2).

Muscle couché à l'entrée du bassin parallèlement au col de 1'ilium et divisé en 
deux portions limitant une gouttière dans laquelle est logée l'extrémité du grand psoas (pl. ro).

- La portion externe, plus volumineuse, est attachée le long du bord externe, sur la face interne et sur l'angle externe de l'ilium.

- La portion interne, plus petite, s'attache au long de la crête iléo-pectinée. A leur extrémité postérieure commune, les deux portions s'attachent par un tendon commun avec celui du grand psoas sur le trochantin du fémur.

48. Tenseur du fascia lata (Tensor fasciae latae) (p1. 7).

Muscle dont le corps charnu, aplati, recouvre en accent circonflexe la région haute des muscles antérieurs de la cuisse. Son extrémité supérieure en pointe s'attache sur l'angle externe de l'ilium. La région inférieure se prolonge par une très large bande aponévrotique : le fascia lata dont on distingue un feuillet superficiel qui s'unit aux aponévroses des régions voisines et notamment à l'aponévrose jambière, et un feuillet profond qui va s'attacher au bord externe du fémur après être passé sous le muscle long vaste.

49. Couturier (Sartorius) (p1. 9-Io).

Long muscle étroit et superficiel de la face interne de la cuisse, étiré entre le droit interne et le vaste interne. Son extrémité supérieure divisée en deux courts faisceaux, est réunie par une bande fibreuse à l'aponévrose lombo-iliaque qui recouvre l'origine du petit psoas. L'extrémité inférieure se continue par une bande aponévrotique commune avec celle du droit interne, et qui a donc les mêmes attaches que cette dernière.

50. Droit interne de la cuisse (Gracilis) (p1. 9-Io).

Large muscle quadrilatère de la face interne de la cuisse, dont l'extrémité supérieure prend attache au long de la symphyse ischio-pubienne suivant une lame aponévrotique commune avec celle du muscle opposé homologue. Dans la région inférieure les fibres se continuent par une large bande aponévrotique unie à celle du couturier et qui prend attache d'une part sur le ligament rotulien interne, d'autre part sur la face interne du tibia où elle contribue à la formation de 1'aponévrose jambière. La partie charnue est assez fortement adhérente aux muscles sous-jacents.

5I. Long vaste (Biceps femoris et abductor cruris cranialis) (p1. 7).

Très important muscle de la région latérale externe de la cuisse, étalé selon une apparence sigmoïde de la région sacrée à l'aponévrose jambière. Il présente dans sa moitié inférieure un faisceau non séparable mais distinct par sa coloration plus pâle, et qui correspond au muscle paraméral d'autres espèces (Lapin, Chat, Porc, Camélidés, d'après Chauveau, ARLoIng et LESBRE). (Ce muscle paraméral est 1'abductor cruris cranialis.)

- L'extrémité supérieure s'insère successivement sur l'épine sacrée, le ligament sacro-sciatique, puis sur la face inférieure de l'ischium, tout autour du tubercule externe de la tubérosité ischiale.

- L'extrémité inférieure, par ses prolongements tendineux aponévrotiques, s'insère successivement sur la rotule, sur le ligament tibio-rotulien du côté externe, puis sur 1'aponévrose jambière ;

L'enlèvement de ce muscle volumineux découvre une série de muscles sousjacents (p1. 8). 


\section{Demi-tendineux (Semitendinosus) (pl. 7, 8, 9, Io).}

Long muscle prismatique de la région postérieure de la cuisse, accolé sur une partie de sa longueur au demi-membraneux, et généralement de coloration nettement plus pâle que ce dernier.

L'extrêmité supérieure se fixe sur le tubercule externe de la tubérosité ischiale. L'extrémité inférieure se continue par une lame aponévrotique qui d'une part va recouvrir la corde du jarret en participant à l'aponévrose jambière et d'autre part va se fixer sur la face médiale de la crète tibiale.

\section{Demi-membraneux (Semimembranosus) (p1. 7, 8, 9, IO, II, I2).}

Très gros muscle de la région postérieure et latérale interne de la cuisse, d'allure prismatique à 3 faces, présentant sur toute sa longueur de gros faisceaux parallèles de fibres.

Son extrémité supérieure s'insère à la face inférieure de l'ischium et présente une pointe qui va s'attacher sous le tubercule externe de la tubérosité ischiatique.

Son extrémité inférieure présente de courts tendons : 1'un, dans la région postérieure, se fixe sur la tubérosité supérieure interne du tibia ; l'autre, au-dessus du ligament fémoro-tibien interne, s'étale sur la face latérale interne de l'extrémité inférieure du fémur où il se fixe, en arrière de la lèvre interne de la trochlée fémorale.

Enfin, plus profondément, l'extrémité antérieure du corps charnu s'insère directement sur la face postéro-inférieure du fémur, immédiatement au-dessus du condyle interne.

\section{Pectiné (Pectineus) (p1. Io, II).}

Muscle conoïde, de texture délicate, aplati à son extrémité inférieure qui se termine par un large tendon aponévrotique aux fibres finement dessinées et qui se fixe sur le corps du fémur, dans sa région postérieure moyenne. L'extrémité supérieure s'attache sur le bord antérieur du pubis par un court tendon qui est commun avec un faisceau de 1'obturateur externe.

55. Adducteur de la cuisse (Adductor brevis et adductor magnus) (p1. 8, II, I2).

Chez le Mouton, le petit adducteur (adductor brevis) et le grand adducteur (adductor magnus) ne sont pas distincts l'un de l'autre et ne forment, par conséquent, qu'un seul muscle adducteur de la cuisse.

C'est un muscle de forme conique, inséré directement à son extrémité supérieure à la face inférieure du pubis, à la face inférieure de l'ischium et sur la lame tendineuse de la symphyse ischio-pubienne.

Son extrémité inférieure s'attache directement sur le corps du fémur dans sa région médiane postérieure où elle s'arrête nettement au-dessus de la dépression intercondylaire.

\section{Fessier moyen (Glutaeus medius) (p1. 7, 8).}

Gros et large muscle qui s'étend de la fin de la région lombaire jusqu'au trochanter du fémur.

Sa région antérieure est fixée sur le bord antérieur de l'ilium, entre les deux angles antérieurs. Elle présente une expansion arrondie adhérente à la fosse iliaque externe et qui déborde en avant sur l'aponévrose du long dorsal. 
L'extrémité postérieure s'attache sur le trochanter du fémur qu'elle enveloppe, et déborde par un fin prolongement en pointe étirée sur l'attache supérieure du vaste externe (p1. 8).

Le bord inférieur du muscle se termine par une expansion fibreuse nacrée qui s'attache le long du bord externe de l'ilium, en recouvrant le bord inférieur du fessier accessoire.

\section{Fessier accessoire (Glutaeus accessorius) (pl. I2).}

Entièrement recouvert par le muscle précédent, c'est un muscle aplati étalé sur la face supérieure de l'ilium où il s'insère directement, de même que sur le bord antérieur de cet os. Il présente dans sa moitié postérieure une aponévrose nacrée se terminant en un large tendon qui s'insère sur le trochanter du fémur, de son sommet antérieur à sa base.

Il recouvre étroitement la portion antérieure du fessier profond, attachée comme lui à la base du trochanter fémoral.

\section{Fessier profond ou Scansorius (Glutaeus profondus) (pl. I3, I5).}

Muscle aplati à faisceaux rayonnants qui convergent en recouvrant le col du fémur vers la base antérieure du trochanter où se fait par un court tendon l'insertion inférieure.

Le corps charnu s'étale en éventail, en s'y insérant, sur la face externe du col de l'ilium et de la région antérieure de l'ischium, et jusque sur le ligament sacro-sciatique.

59. Jumeaux du bassin (Mm. gemelli) (pl. 8, I2, I3).

Chez le Mouton, ils correspondent à un seul petit muscle de la région postéroinférieure du bassin, situé entre le scansorius et les muscles obturateurs.

Dans sa région supérieure il se fixe sur la face inférieure de l'ischium en avant du tubercule externe de la tubérosité ischiale; 1'extrémité inférieure se termine par un tendon commun avec celui des obturateurs, dans la fosse trochantérienne.

6o. Carré fémoral (Quadratus femoris) (p1. 8, I2, I3, I5).

Petit muscle rectangulaire s'étendant du trochantin du fémur à la face inférieure de l'ischium, en avant de la tubérosité ischiale.

6r. Obturateurs (Obturatorius externus et Obturatorius internus) (p1. I2, I3, I5).

Les muscles obturateurs ont un tendon inférieur commun qui s'insère dans la fosse trochantérienne du fémur, et sont assez difficiles à isoler l'un de l'autre. Ils sont séparables à un niveau qui correspond au bord pubien du trou ovalaire de l'os coxal.

L'obturateur externe (Obturatorius externus) prend attache sur la face inférieure du pubis et de la jonction ischio-pubienne.

I'obturateur interne (Obturatorius internus) présente une particularité remarquable. Par ses faisceaux externes, qui font suite à l'obturateur externe, il s'attache également à la face inférieure du pubis et surtout de l'ischium; et par ses faisceaux profonds il pénètre dans le trou ovalaire pour aller s'attacher sur presque tout le pourtour de celui-ci, sur le plancher même du bassin. 
62. Vaste externe (Vastus lateralis) (p1. 8, Io).

Muscle large et épais de la face latérale externe de la cuisse. Fixé postérieurment sur le trochanter et le bord externe du fémur, il se termine en avant sur la rotule. II recouvre la région correspondante $d u$ vaste intermédiaire et il adhère très fortement à l'aponévrose d'enveloppe du droit antérieur qu'il recouvre également sur sa face externe (Par rapport à la pl. 8, la pl. I2 est figurée après enlèvement de ce muscle.)

\section{Droit antérieur de la cuisse (Rectus femoris) (p1. IO, II, I2).}

Gros muscle de la région antérieure de la cuisse, en forme de fuseau épais et enclavé entre les muscles vastes de la cuisse.

Attaché comme ces derniers sur la rotule par son extrémité inférieure, son extrémité supérieure comporte un court mais fort tendon aplati qui se dédouble pour se fixer à cheval à la base du bord externe de l'ilium, immédiatement en avant de la cavité cotyloïde.

64. Vaste interne (Vastus medialis) (p1. 9-ro-I2).

Muscle large et aplati de la région latérale interne de la cuisse, recouvrant en y adhérant fortement le vaste intermédiaire et 1'extrémité inférieure du droit antérieur.

Son extrémité inférieure est attachée sur la rotule. L'extrémité supérieure est attachée dans la région supérieure du fémur, le long du bord interne ainsi que sous le trochin et sous la tête articulaire qu'elle contourne.

\section{Vaste intermédiaire (Vastus intermedius) (pl. II, I2).}

Muscle formé de 2 faisceaux symétriques accolés chacun sur l'une des faces latérales du fémur sur laquelle il s'insère directement, et réunis dans leur région médiane pour s'insérer sur la partie supérieure de la face antérieure du fémur. Chaque faisceau est recouvert inférieurement d'une belle aponévrose nacrée qui donne naissance à un court tendon s'insérant sur la face latérale correspondante de la rotule.

\section{Articulaire du genou (Articularis genus) (pl. 13, 15).}

Petit muscle recouvert par la partie inférieure des branches du vaste intermédiaire. Il est attaché au sommet de la rotule, de l'angle interne à l'angle externe, et on peut y distinguer trois faisceaux (deux latéraux et un médian), qui convergent en pointe à la base de la face antérieure du fémur, au-dessus de la trochlée.

\section{Soléaire (Soleus) (p1. 8).}

Fine bandelette musculaire allongée, de la région externe de la jambe. Son extrémité supérieure est attachée au ligament fémoro-tibien externe. Ce petit muscle s'insinue ensuite sous le jumeau externe au sein duquel vont se perdre les fibres de son extrémité inférieure, vers la base de celui-ci et sur une lame tendineuse interne.

\section{Jumeaux de la jambe (Gastrocnemius) (p1. 8, Io, II, I2).}

C'est un puissant muscle de la région postérieure de la jambe, qu'on peut dissocier en deux faisceaux jumeaux, entourant le muscle planto-perforé, et unis sur la ligne postérieure médiane de la jambe. Ces faisceaux ont d'ailleurs un fort tendon 
commun inférieur qui va s'attacher sur le sommet du calcanéum après avoir longé celui du planto-perforé (sur la pl. I2, face externe de la jambe, on aperçoit le jumeau interne et le planto-perforé après enlèvement du jumeau externe).

Le jumeau interne est attaché antérieurement à la base du fémur, au-dessus du condyle interne.

Le jumeau externe est attaché symétriquement, au-dessus du condyle externe, sur le bord externe de la fosse sus-condylienne.

\section{Planto-perforé (Flexor digitorum superficialis) (p1. IO, II, I2, I3, I4, I5)..}

Muscle fusiforme encadré par les jumeaux de la jambe, et fortement fixé dans sa région supérieure à la base du fémur, dans la fosse sus-condylienne.

L'extrémité inférieure se continue par un fort et très long tendon qui se réfléchit sur le sommet đu calcanéum avant de descendre le long de la face postérieure de l'os canon à la base duquel il se dédouble en deux branches. Chacune de ces branches, avant d'aller s'attacher à l'extrémité supéro-postérieure de la $2^{\mathrm{e}}$ phalange, forme un anneau à l'intérieur duquel coulisse la branche correspondante du fléchisseur latéral des doigts (" perforant").

\section{Long péronier (Peroneus longus) (p1. I3).}

Muscle triangulaire allongé de la face externe de la jambe, à pointe dirigée vers le bas. A son extrémité supérieure le corps charnu se fixe à la base de la tubérosité supérieure externe du tibia. Le mince tendon inférieur descend le long de la face inférieure externe du tibia, puis traverse obliquement la région tarsienne pour aller s'attacher dans la région postéro-inférieure du tarse, immédiatement au-dessus de l'os canon, sur l'os petit-cunéiforme.

7I. Masse commune de la jambe (Extensor digitorum longus) (pl. I3, I5). (Les N.A.V. ne donnent pas de nom particulier aux subdivisions.)

Muscle complexe de la région antérieure de la jambe, formé par trois faisceaux distincts dans leur partie inférieure, et réunis à leur extrémité supérieure où ils se prolongent par un fort tendon qui vient se fixer à la base du fémur, entre la lèvre externe de la trochlée et le condyle externe, après avoir glissé dans la coulisse tibiale.

- Le faisceau antérieur principal, plus large et enveloppant partiellement les deux autres, se prolonge à son extrémité inférieure par un fort tendon qui vient s'insérer en fourche d'une part à l'extrémité supéro-antérieure de l'os canon et d'autre part sur le bord postérieur du grand cunéiforme. Il correspond au muscle fléchisseur du pied.

Les deux autres faisceaux, plus petits et fusiformes, sont d'importance égale. Le faisceau interne correspond au muscle extenseur propre du doigt interne. Le faisceau externe correspond au muscle extenseur commun des doigts. Les extrémités inférieures des tendons de ces deux faisceaux se comportent comme celles des muscles correspondants de 1'avant-bras ( $\mathbf{n}^{\circ} 93$ et $\mathrm{n}^{\circ} 95$ ).

\section{Extenseur propre du doigt externe (Extensor digitorum latèralis) (p1. I3).}

Muscle fusiforme de la région latérale externe de la jambe, très adhérant au fléchisseur latéral des doigts sur la plus grande partie de sa longueur. Son court tendon supérieur est fixé sur le bord latéral de la surface articulaire supérieure externe du 
tibia, ainsi qu'à la base du ligament fémoro-tibial externe. Les fibres charnues adhèrent à une aponévrose d'enveloppe du fléchisseur latéral des doigts ainsi qu'au cordon fibreux qui figure le péroné. Le long tendon inférieur va se fixer au sommet antérieur de la deuxième phalange du doigt externe, après avoir longé le profil externe des régions inférieures de la jambe et du pied

\section{Tibial cranial (Tibialis cranialis) (p1. I3, I4, I5).}

Long muscle aplati triangulaire logé dans la fosse antéro-externe du tibia au sommet de laquelle il se fixe. Son tendon inférieur longe la région antéro-interne inférieure du tibia, puis traverse obliquement la région tarsienne après avoir traversé une échancrure du tendon du fléchisseur du pied, pour venir s'attacher à l'extrémité supérieure de l'os canon, du côté interne, à la base des os cunéiformes.

\section{Tibial caudal (Tibialis caudalis) (pl. 13, 14, 15).}

Muscle de la région postérieure de la jambe, appliqué contre le bord postérieur du fléchisseur latéral des doigts auquel il adhère légèrement dans sa partie haute. Il est d'ailleurs fixé avec ce dernier, par son extrémité supérieure, sur la tubérosité supéro-externe du tibia. Le tendon inférieur va également se confondre avec celui du fléchisseur latéral des doigts dans la région postéro-inférieure du corps du tibia.

\section{Fléchisseur latéral des doigts (Flexor hallucis longus) (p1. I3, I4, I5).}

Le plus important des muscles fléchisseurs de la jambe. Son corps charnu est plaqué.sur la face postérieure du tibia, à laquelle il adhère sur presque toute sa longueur.

L'extrémité supérieure est fixée à la tubérosité supéro-externe du tibia, ainsi qu'au cordon fibreux figurant le péroné.

Son fort tendon inférieur coulisse sur la saillie interne du calcanéum après avoir reçu le tendon du tibial caudal et avant de recevoir celui du fléchisseur médial des doigts. Il longe ensuite la face postérieure de l'os canon à la base duquel il se dédouble en deux branches, une pour chaque doigt, chacune allant s'insérer sous la $3^{\mathrm{e}}$ phalange correspondante.

\section{Fléchisseur médial des doigts (Flexor digitorum longus) (p1. I5).}

Muscle de la région postérieure de la jambe, situé entre le poplité et lefléchisseur latéral des doigts auquel il adhère légèrement.

L'extrémité supérieure du corps charnu, assez court, se fixe par un court tendon sur le bord postérieur de la surface articulaire supérieure externe du tibia.

Le tendon inférieur, après avoir traversé dans une gaine fibreuse la région interne du tarse, va se confondre avec le tendon du fléchisseur latéral des doigts au niveau de la région postéro-supérieure de l'os canon.

\section{Poplité (Popliteus) (p1. I3, I4, I5).}

Muscle d'aspect triangulaire de la face postérieure de la jambe, entourant d'un bord à l'autre l'extrémité supérieure de la face postérieure du tibia. Il présente un tendon et un corps charnu.

- Le tendon, après être passé sous le ligament fémoro-tibial externe, s'insère dans la fossette inférieure du condyle externe du fémur. 


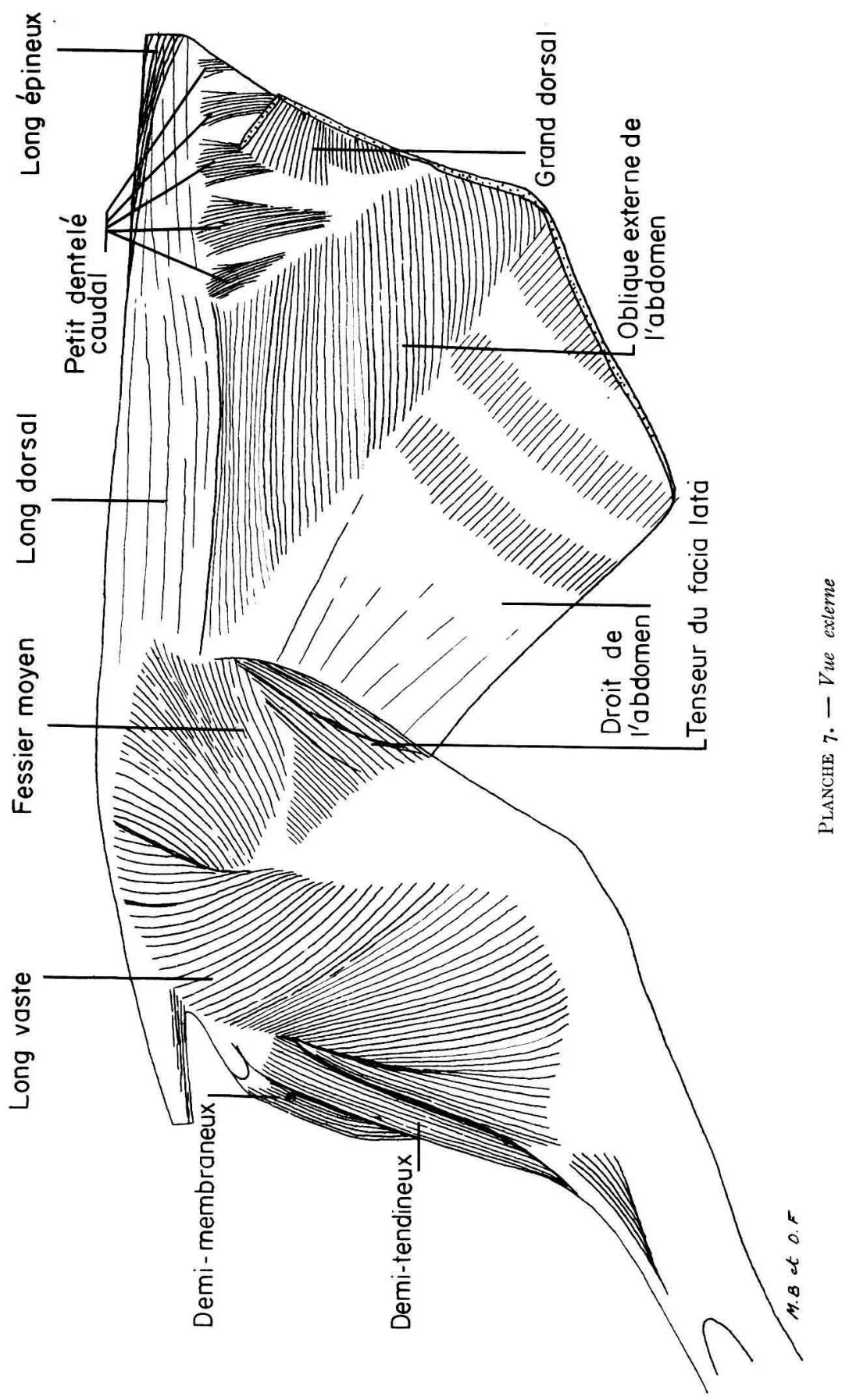




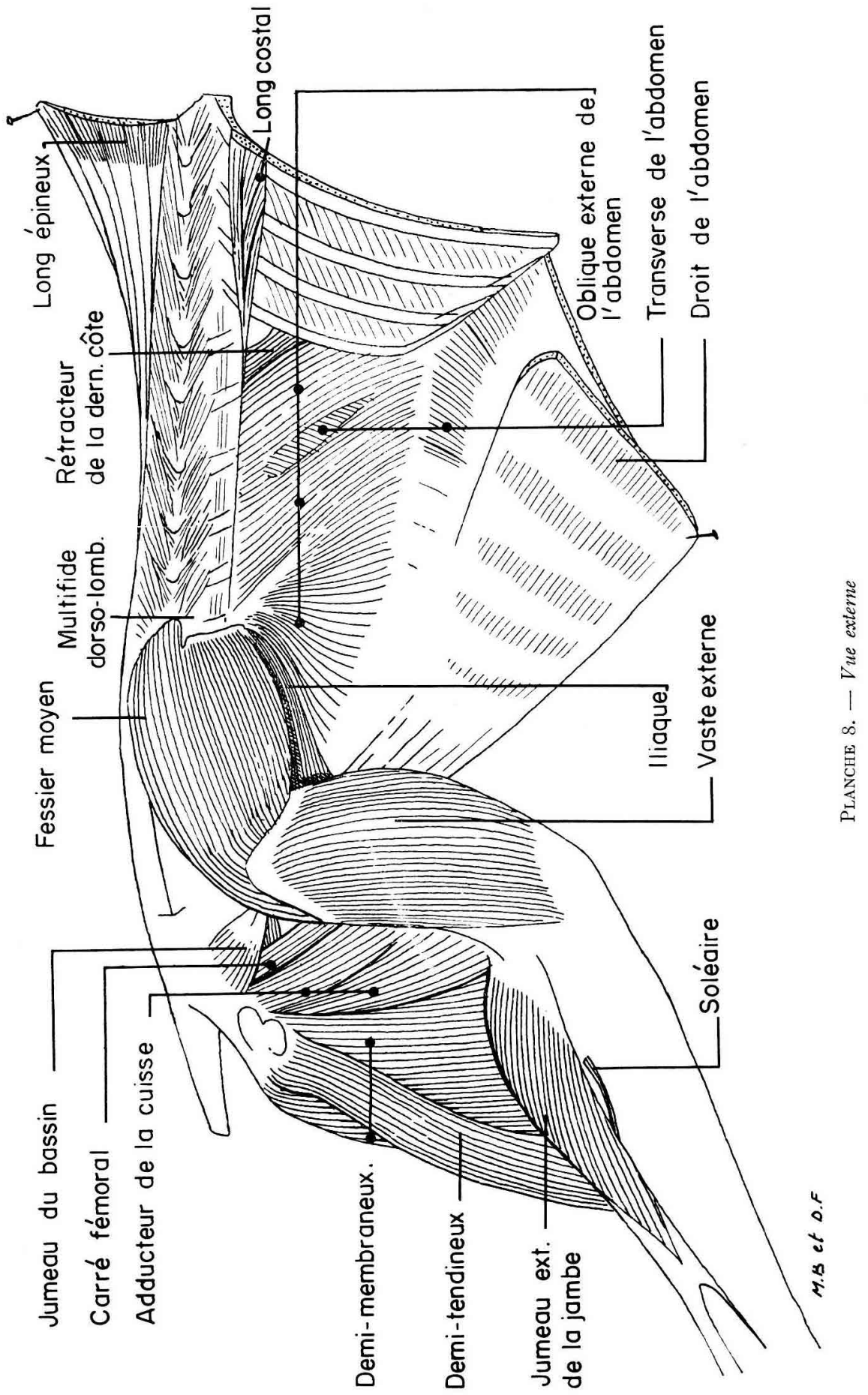




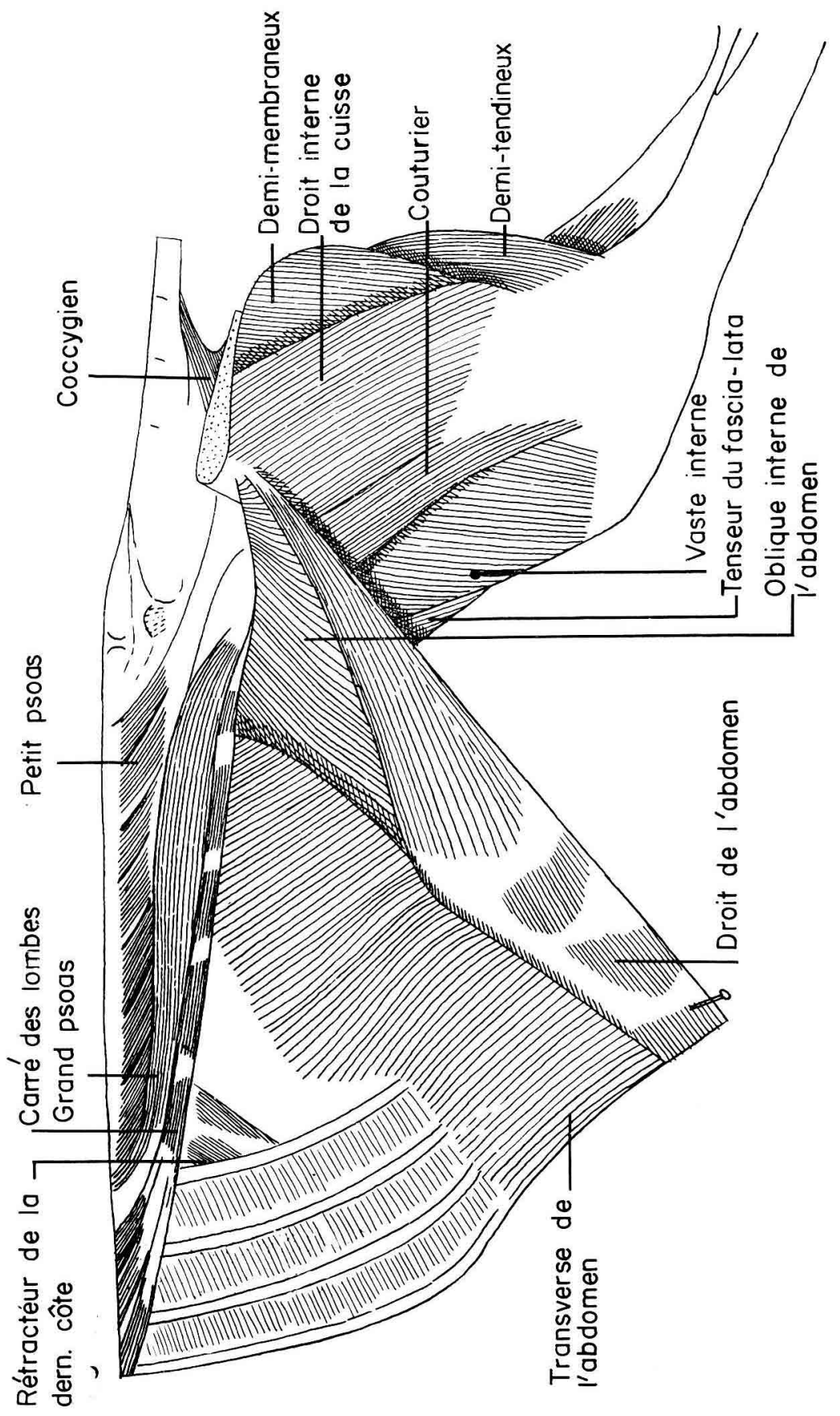

焉

4
0
3
5 


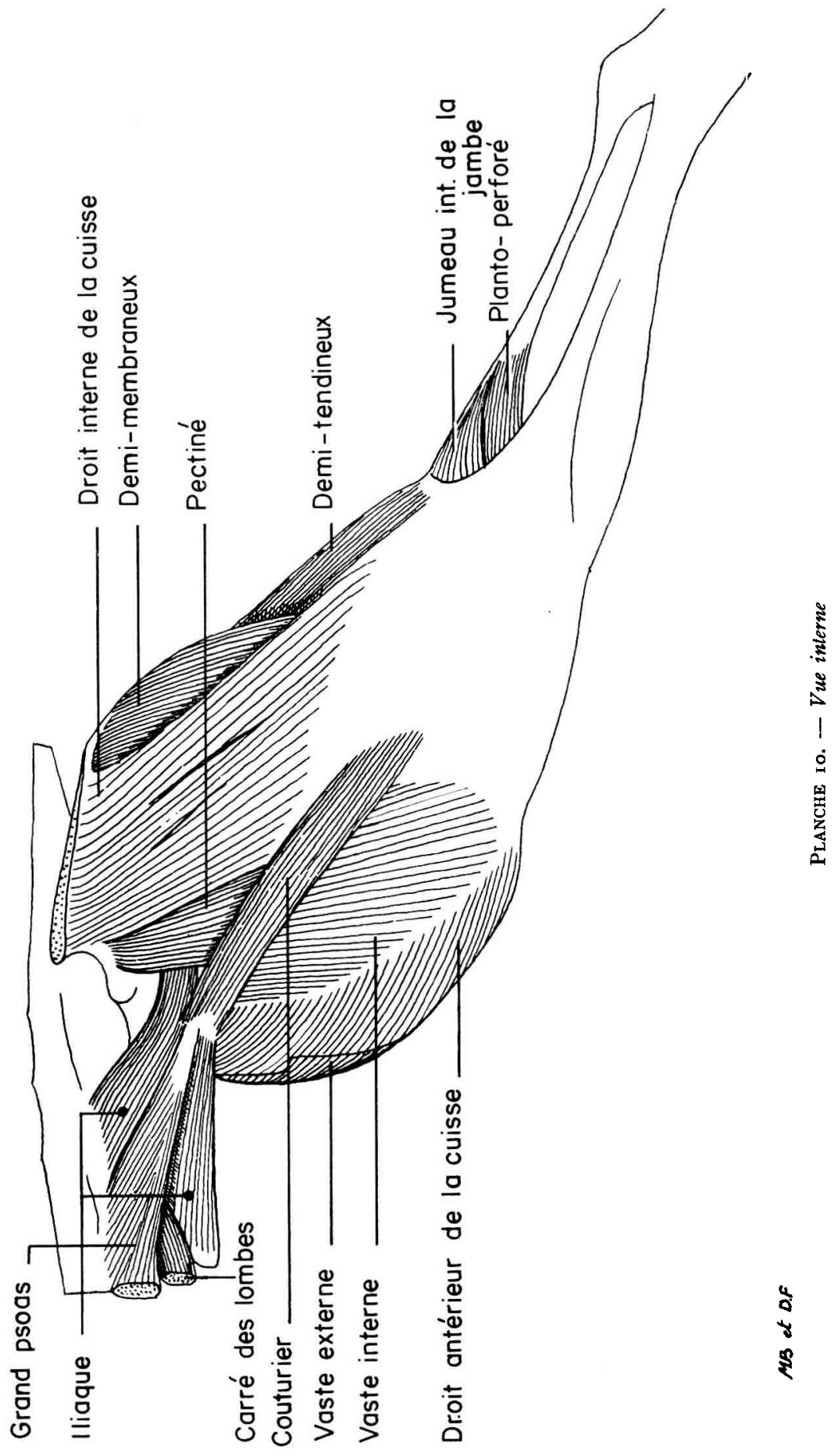




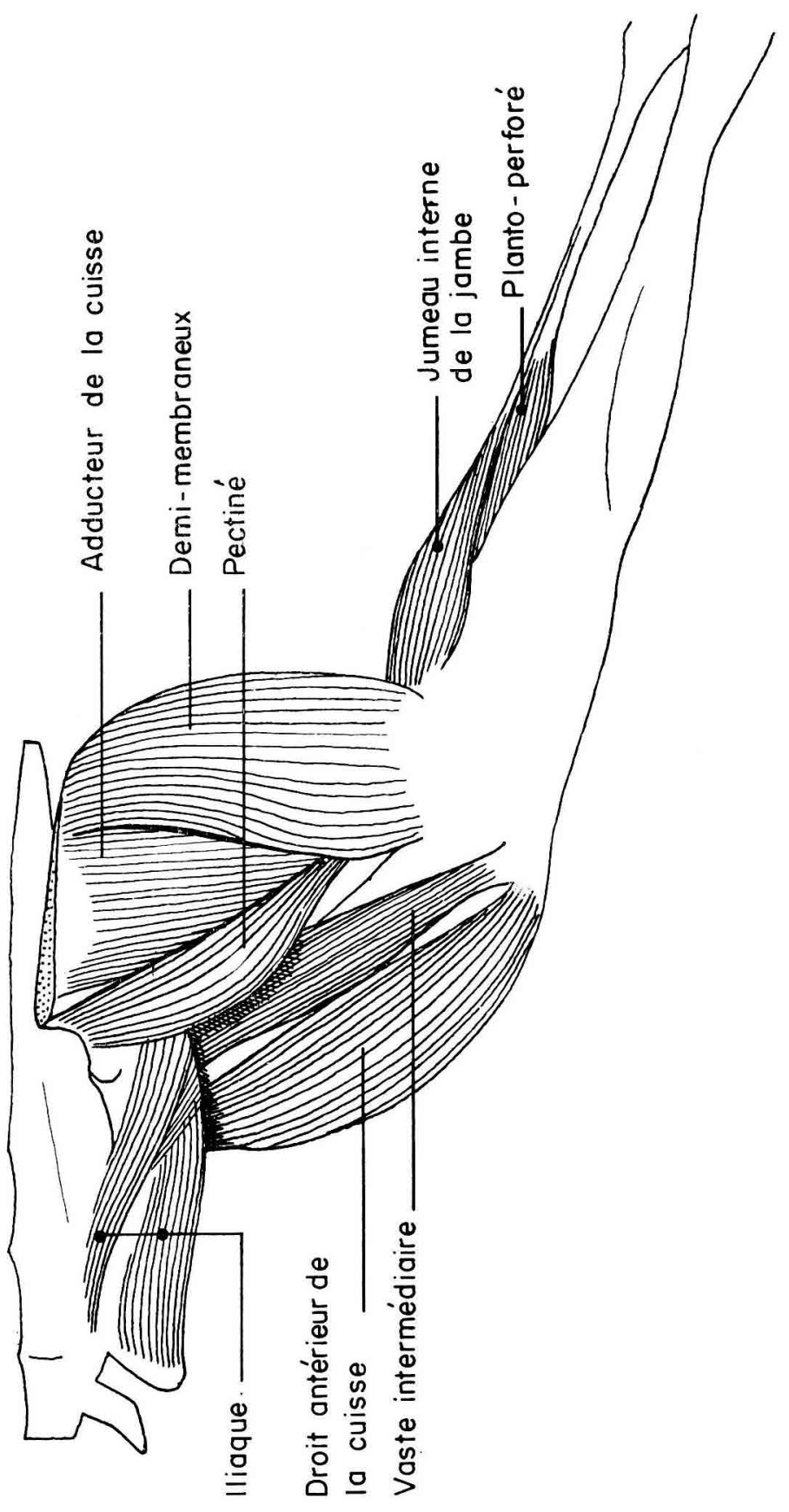




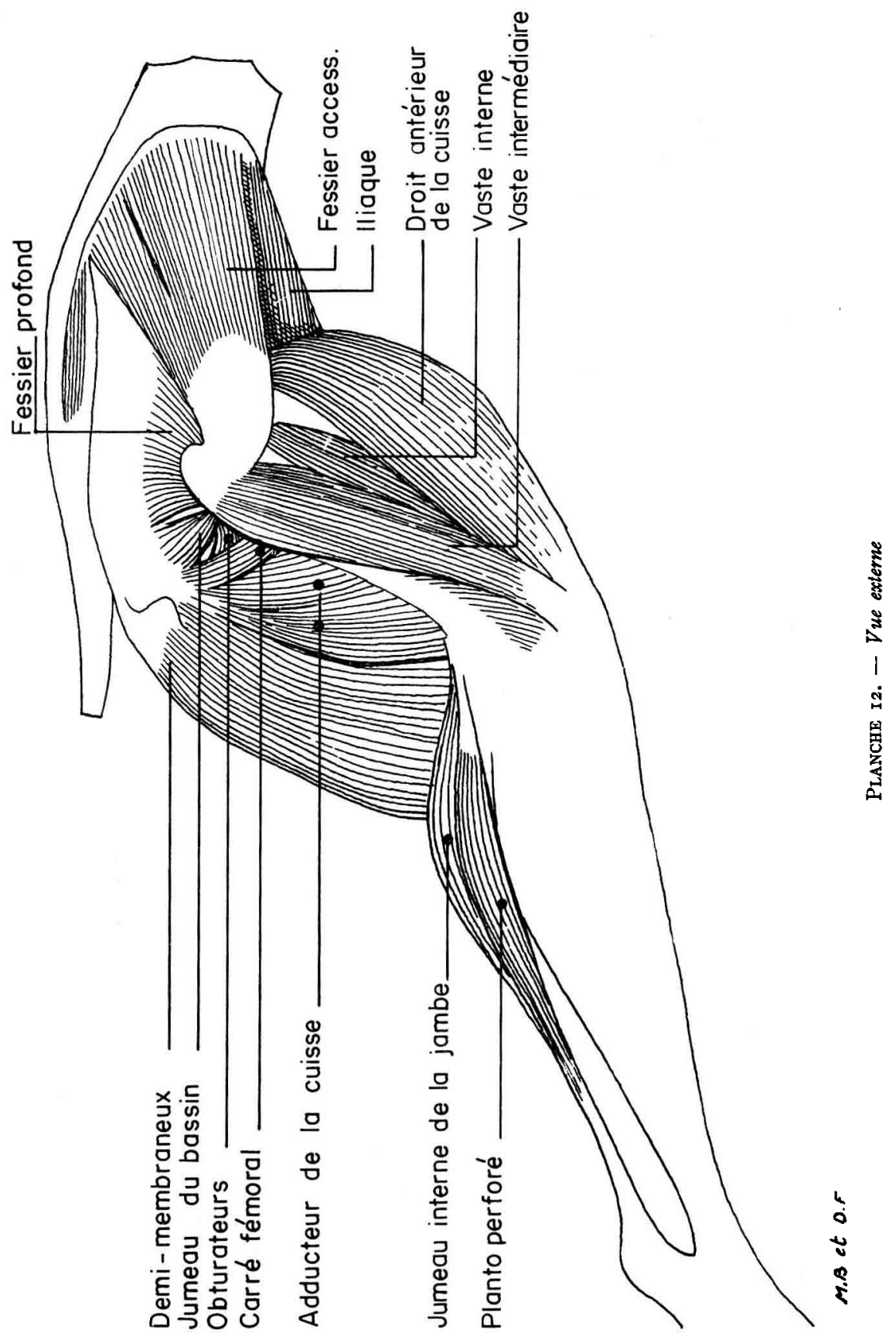




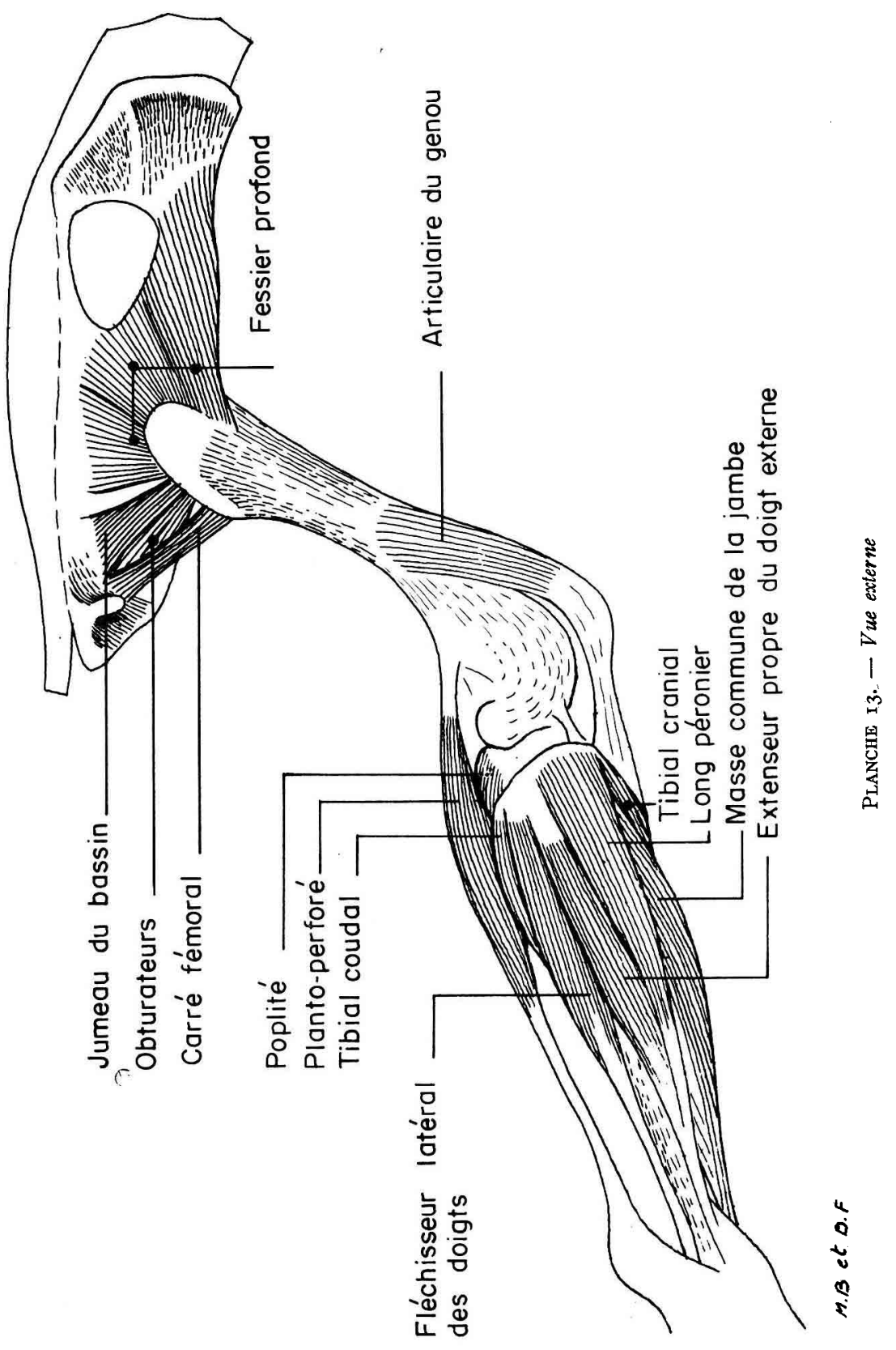




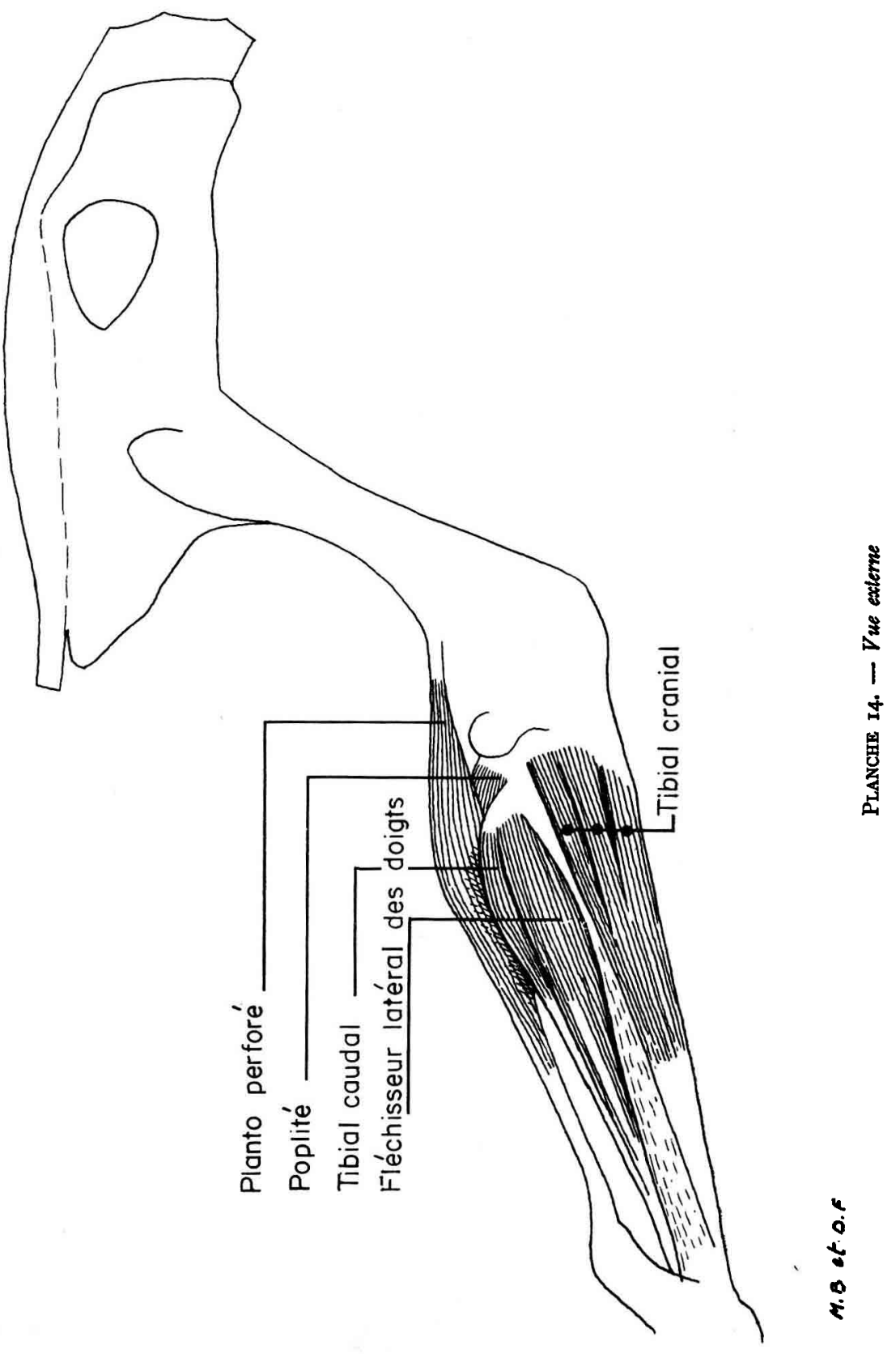




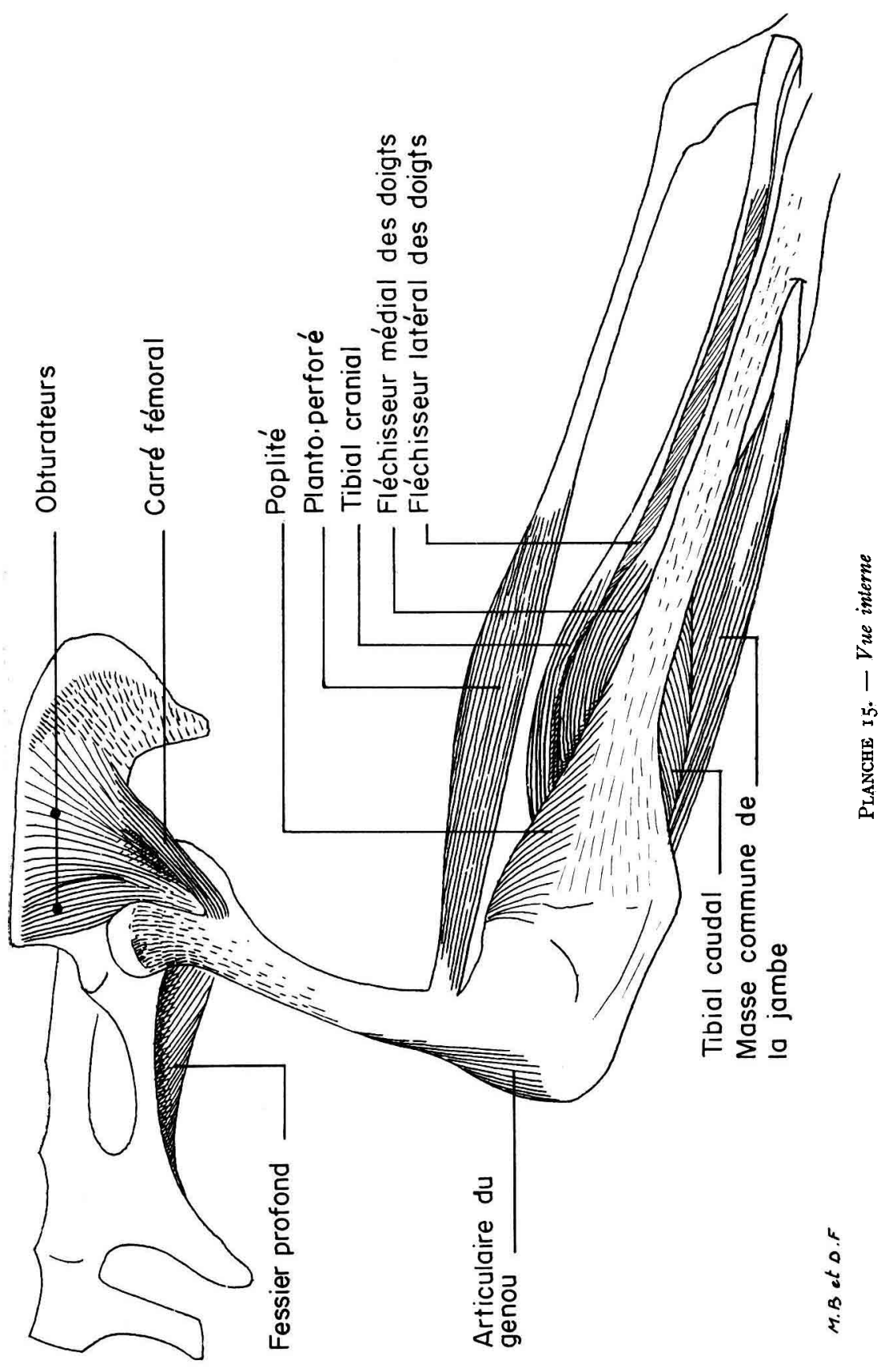


- Le corps charnu adhère au bord latéral interne du tibia dans son tiers supérieur, ainsi que sur la surface triangulaire supérieure interne de la' face postérieure du tibia.

\section{III. - MEMBre ANTÉRIEUR (pl. I6 à 20)}

78. Deltoide (Deltoideus) (p1. I6).

Muscle bifide de la base de la face externe de l'épaule; ses deux faisceaux entourent l'extrémité inférieure du muscle sous-épineux contre lequel ils sont plaqués.

- Le faisceau antérieur, le plus long, s'attache à sa base, qui s'étire en pointe, en haut de la crête humérale ; son extrémité supérieure se fixe à la base de l'épine acromienne du scapulum ainsi que sur l'aponévrose scapulaire qui recouvre le sousépineux.

- Le faisceau postérieur, logé dans une dépression comprise entre les muscles anconés et le sous-épineux, est lui-même fixé dans sa partie haute à l'aponévrose scapulaire, et son extrémité inférieure rejoint par une lame aponévrotique le corps du faisceau antérieur, au tiers inférieur de celui-ci.

L'enlèvement de ce muscle découvre notamment l'extrémité inférieure du muscle petit rond (p1. I7).

79. Sous-épineux (Infraspinatus) (p1. 16, I7).

Large muscle de la face externe de l'épaule, occupant entièrement la fosse sousépinetuse du scapulum à laquelle il adhère fortement, et débordant largement sur le cartilage de prolongement auquel les fibres de l'extrémité supérieure adhèrent également.

L'extrémité inférieure se poursuit par un fort et large tendon qui va s'attacher à la base du trochiter huméral.

8o. Sus-épineux (Supraspinatus) (p1. 16, I7, I8).

Muscle important de la région antérieure de l'épaule, dont la majeure partie occupe la fosse sus-épineuse en y adhérant fortement, de même qu'au bord antérieur de la palette du scapulum.

L'extrémité inférieure, fixée postérieurement au bord inférieur de l'épine acromienne, entoure d'autre part entièrement la région antérieure de l'articulation scapulohumérale, s'attachant sur le rebord du trochiter puis du trochin en passant pardessus le tendon supérieur du biceps logé dans sa coulisse bicipitale. Il existe aussi une légère attache sur l'apophyse coracoïde.

8r. Petit rond (Teres minor) (p1. I7, I9).

Petit muscle de la région scapulo-humérale postérieure externe, situé en dessous et en arrière de l'extrémité inférieure du sous-épineux. Sa région supérieure se fixe par une série de languettes tendineuses le long du bord postérieur du scapulum, depuis le tiers inférieur jusqu'au bord de la cavité glénoïde. Son extrémité inférieure s'attache par un court tendon à la base du bord postérieur du trochiter huméral.

82. Sous-scapulaire (Subscapularis) (p1. I8).

Large muscle étendu sur la face interne du scapulum en trois faisceaux distincts dont le plus important, le faisceau médian, s'étale en éventail jusque sur le cartilage 
de prolongement de l'os. Les faisceaux latéraux, plus courts, sont attachés respectivement le long du bord antérieur et du bord postérieur du col du scapulum. L'ensemble s'attache, à l'extrémité inférieure, par un très fort tendon commun, sur la convexité du trochin de l'humérus.

Pour sectionner cette dernière attache, il est utile de sectionner auparavant le tendon d'insertion du muscle coraco-brachial sur l'apophyse coracoïde.

83. Grand rond (Teres major) (p1. I6, I7, r8, I9, 20).

Muscle allongé le long du bord postérieur du scapulum, à l'angle dorsal duquel il s'attache. Sa partie moyenne est étroitement accolée au faisceau postérieur du muscle sous-scapulaire, et son extrémité inférieure vient s'insérer à la face interne de 1'humérus, vers le tiers antérieur.

\section{Coraco-brachial (Coracobrachialis) (p1. I8).}

Muscle dont les fibres s'attachent en fuseau allongé sur la moitié antérieure de la face interne du corps de l'humérus, et dont l'extrémité antérieure vient se fixer, par un tendon aplati, sur l'apophyse coracoïde, en recouvrant le tendon d'insertion inférieur du sous-scapulaire.

85. Anconé accessoire du grand dorsal (Tensor fascia antebrachii) (p1. I6, I7, I8).

Etroite bande charnue reliant l'angle dorsal du scapulum, près de l'insertion supérieure du muscle grand rond, à la face interne et au bord postérieur de l'olécrâne, en longeant le bord postérieur du muscle long anconé.

\section{Long anconé (Caput longum tricipitis brachii) (p1. I6, I7, I8).}

Volumineux et puissant muscle triangulaire, logé dans 1'angle scapulo-huméral dont il occupe la plus grande partie. Son extrémité inférieure comporte une lame tendineuse qui se termine en un fort tendon enveloppant les régions supérieures de l'olécrane sur lesquelles il se fixe. Il est inséré d'autre part, à sa partie supérieure, tout au long du bord postérieur du scapulum.

\section{Anconé externe (Caput laterale tricipitis brachii) (p1. I6-I7).}

Muscle large allongé selon la ligne de l'humérus. Son extrémité postérieure est insérée par un court tendon sur la face supéro-externe de l'olécrane. Son extrémité antérieure s'insère, en arrière de la tête articulaire de l'humérus et latéralement, sur la ligne courbe allant de la tête articulaire à la tubérosité deltoïdienne.

\section{Anconé interne (Caput mediale tricipitis brachii) (p1. I8, I9, 20).}

Muscle aplati d'aspect triangulaire, dont l'extrémité postérieure tendineuse est fixée à la face interne de l'olécrane. Il s'attache d'autre part à la face interne du corps de l'humérus, sur sa moitié antérieure.

De ce muscle se détache, à la partie supérieure, une mince bandelette musculaire isolable sur presque toute sa longueur, et reliant 1'olécrane à la base de la tête articulaire de l'humérus (pl. 20). Cette bandelette, qui paraît faire partie de l'anconé interne car issue du même tendon olécranien, semble particulière au mouton et a parfois été décrite sous le nom de Anconé profond ou anconé moyen (CHaUvEaU, ARLOING et LESBRE, Ig.03) (Caput accessorium tricipitis brachii). 
89. Petit anconé (Anconceus) (p1. Ig).

Petit muscle tendu au-dessus de la fosse olécranienne, inséré d'une part sur la face externe de l'olécrane, d'autre part sur le corps de 1'humérus dans sa moitié supéro-postérieure.

90. Biceps brachial (Biceps brachii) (p1. 16, 18, 19, 20).

Muscle en fuseau régulier, étiré le long de la face antérieure de l'humérus.

Le tendon supérieur, fortement allongé, glisse sur la coulisse bicipitale de l'humérus et vient s'attacher sur 1'apophyse coracoïde du scapulum.

- Le tendon inférieur se subdivise en deux faisceaux : l'un court, s'attache dans la région antéro-supérieure du radius ; l'autre, plus long, contourne le radius par son bord interne pour aller s'attacher sur le cubitus.

9x. Brachial (Brachialis) (p1. I6, I8, I9, 20).

Muscle allongé, d'allure rectangulaire, logé dans la gouttière de torsion de l'humérus qu'il contourne d'arrière en avant par l'extérieur.

Il prend origine en arrière de la tête articulaire et du trochiter de l'humérus. Il se termine, comme le biceps, par detux branches tendineuses analogues, dont les insertions sont situées immédiatement au-dessous des insertions correspondantes du biceps.

\section{Extenseur radial du carpe ou Extenseur antérieur du métacarpe (Extensor carpi radialis, longus et brevis) (p1. I6-18).}

Forte masse musculaire fusiforme longeant la face antérieure du radius dont elle recouvre largement la moitié supérieure.

Elle présente dans sa partie supérieure deux régions d'insertion à la base de l'humérus : l'une sur le bord externe de l'os, l'autre immédiatement au-dessus de la gorge de la trochlée articulaire.

L'extrémité inférieure se prolonge par un fort tendon aplati qui glisse en avant des os du carpe pour aller s'attacher sur la tubérosité antéro-interne de l'os canon.

93. Extenseur propre du doigt interne (p1. I6, I9) (comme dans la jambe, muscle spécial aux ruminants, non nommé dans les $N . A . V$.)

Muscle fusiforme adhérant dans sa partie supérieure à l'extenseur antérieur du métacarpe. Son extrémité supérieure s'insère par un court tendon à la base de la face antérieure de 1'humérus, immédiatement au-dessus du condyle externe. Le tendon inférieur passe d'abord dans la coulisse inférieure centrale légèrement externe du radius, puis croise obliquement l'os canon pour venir finalement s'attacher au sommet antérieur de la deuxième phalange du doigt interne.

\section{Extenseur propre du doigt externe (Extensor digitorum lateralis) (p1. I6, I9).}

Son corps charnu se divise dans sa partie supérieure en deux courtes branches, l'une attachée sur la tubérosité supéro-externe du radius, l'autre remontant jusqu'à la base de la crête postérieure de la gouttière de torsion de l'humérus, où elle prend attache conjointement avec une des branches de l'extenseur commun des doigts, immédiatement au-dessus de l'excavation ligamenteuse. D'autre part, le corps du 
muscle est attaché, dans sa partie moyenne, au long đu bord postérieur du cubitus.

Le tendon inférieur passe dans la coulisse la plus externe du bord inférieur du radius, et après avoir longé l'os canon va s'attacher au sommet antérieur de la deuxième phalange du doigt externe.

\section{Extenseur commun des doigts (Extensor digitorum communis) (p1. I6, I9).}

Situé entre les deux muscles précédents, son corps charnu présente deux branches distinctes. La branche la plus profonde se loge dans sa partie supérieure dans l'angle radio-cubital externe, où elle s'insère au-dessus de l'arcade radio-cubitale. L'autre branche s'attache au-dessus de l'excavation ligamenteuse de l'humérus, en commun avec une des branches de l'extenseur propre du doigt externe.

Le tendon inférieur accompagne d'abord celui de l'extenseur propre du doigt interne, puis franchit la partie médiane antérieure de l'os canon, au niveau de laquelle il se dédouble, chacun des deux tendons résultants allant s'attacher sur l'éminence pyramidale de la troisième phalange correspondante.

\section{Cubital externe (Extensor carpi ulnaris) (p1. I6).}

Fort muscle aplati de la région postéro-latérale externe de l'avant-bras. Son extrémité supérieure s'attache sur l'épicondyle de l'humérus.

Le tendon inférieur aplati se divise en deux branches : l'une rejoint le tendon du cubital interne avec lequel elle s'attache en commun sur 1'os sus-carpien; 1'autre descend jusqu'à l'extrémité supérieure de l'os canon sur laquelle elle s'attache, au niveau de la tête du métacarpien latéral rudimentaire externe.

\section{Grand palmaire (Flexor carpi radialis) (p1. $\mathrm{I} 8,20)$.}

Muscle aplati et régulièrement fuselé, situé entre le cubital interne et le cubital externe.

Sa partie charnue, qui correspond à la moitié supérieure du radius, rejoint à son extrémité supérieure le tendon du cubital interne pour s'attacher au niveau de l'épitrochlée de 1'humérus.

Le tendon inférieur s'engage dans une longue gaine fibreuse et va s'insérer à l'extrémité supérieure de l'os canon, au niveau de la tête du métacarpien latéral rudimentaire interne.

\section{Cubital interne (Flexor carpi ulnaris) (pl. I8, 20).}

Muscle aplati recouvrant largement le fléchisseur superficiel des doigts auquel il est partiellement mais fortement adhérent. Son tendon inférieur s'attache sur l'os sus-carpien à côté de l'une des branches du cubital externe. Un court et fort tendon attache l'extrémité supérieure à la base de l'épitrochlée humérale. D'autre part l'extrémité supérieure lance une mince expansion fibreuse qui recouvre le chef olécranien du fléchisseur profond des doigts avant de s'attacher dans la région postérieure de l'olécrane.

\section{Fléchisseur superficiel des doigts (Flexor digitorum superficialis) (pl. I8, I9, 20)}

Situé sous le cubital interne, il s'attache comme lui par son extrémité supérieure, sur l'épitrochlée humérale, comme d'ailleurs aussi un tendon du fléchisseur profond des doigts. 
Le corps charnu se divise en son milieu en deux branches distinctes se prolongeant chacune par un tendon inférieur dont le plus profond est logé dans une gouttière formée par le tendon du fléchisseur profond des doigts. Ces deux tendons dufléchissseur superficiel des doigts se rejoignent d'abord vers le milieu de l'os canon, formant alors une tige unique qui se dédouble ensuite à son tour au niveau des os grands sésamoïdes en une branche pour chaque doigt. Chacune de ces branches se comporte comme celle du tendon du muscle correspondant du membre postérieur (no 69).

I00. Fléchisseur profond des doigts (Flexor digitorum profondus) (pl. 16, 18, 19, 20).

Muscle constitué par trois corps charnus bien différenciés, et dont les tendons inférieurs s'unissent en un très fort tendon commun. On distingue ainsi : un chef huméral, un chef olécranien, un chef radial.

Le chef huméral, médian, le plus volumineux, s'attache par sa partie supérieure sur l'épitrochlée humérale. Il est lui-même constitué de deux faisceaux assez aisément séparables : un faisceau principal qui représente la plus grande part et qui donne naissance à la plus forte partie du tendon inférieur commun ; un petit faisceau secondaire, mince ruban fusiforme qui se distingue notamment de la masse principale par son extrémité inférieure dont un court tendon propre vient se fixer sur le tendon commun.

Le chef olécranien comprend une portion charnue supérieure, de forme pyramidale, qui entoure le bord postérieur de l'olécrane, où elle s'attache de même que sur la face externe et principalement sur toute la face interne de l'olécrane. Il se prolonge par un tendon qui va rejoindre le tendon commun à la base du corps charnu du chef huméral.

Le chef radial, le plus profond et le plus réduit, présente un corps charnu plaqué contre la région supérieure de la face postérieure du radius, et qui se poursuit par un mince tendon qui va rejoindre le tendon principal du chef huméral à la base du corps charnu de ce dernier.

- Le tendon commun, très puissant, longe la face postérieure du carpe puis celle de l'os canon, et au niveau du boulet se divise en deux branches, une pour chaque doigt. Chacune de ces branches après avoir traversé l'anneau correspondant du tendon du fléchisseur superficiel des doigts, va s'insérer sous la troisième phalange après renvoi sur les petits sésamoïdes.

\section{Ior. Extenseur oblique du métacarpe (Abductor pollicis longus et extensor pollicis} brevis) (p1. I6).

Une fois enlevés tous les muscles précédents de l'avant-bras, on distingue encore, plaquée contre le bord externe du radius, dans l'angle radio-cubital, une mince bandelette musculaire qu'on peut interpréter comme représentant le muscle extenseur oblique du métacarpe, atrophié chez le Mouton.

Cette bandelette, à son extrémité inférieure, croise obliquement la face antérieure du radius, puis s'étire jusqu'au métacarpe en un frêle tendon qui ne peut, pratiquement, assurer un rôle actif. 


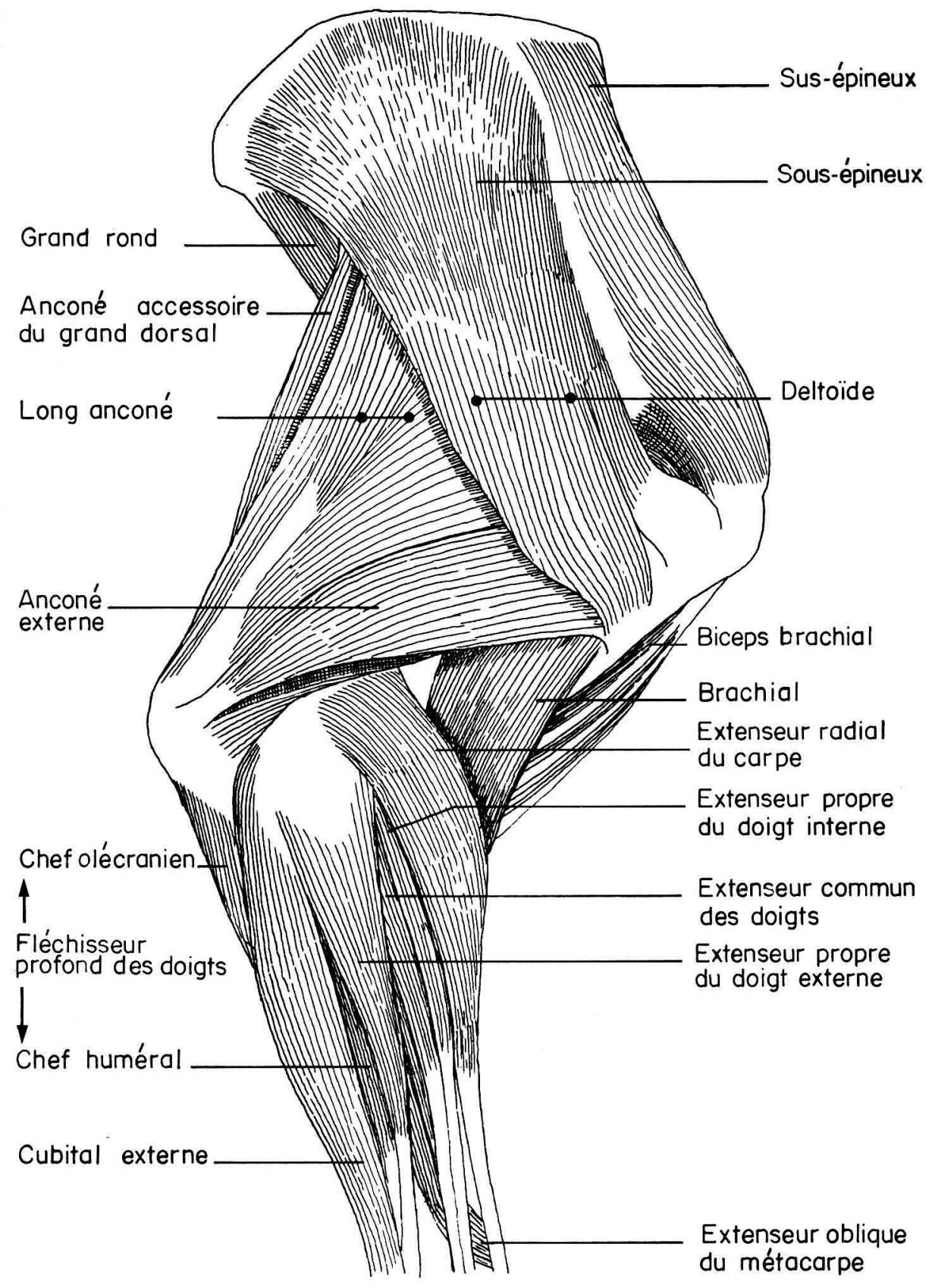

M.B et D.F

Planche 16. - Vue externe 


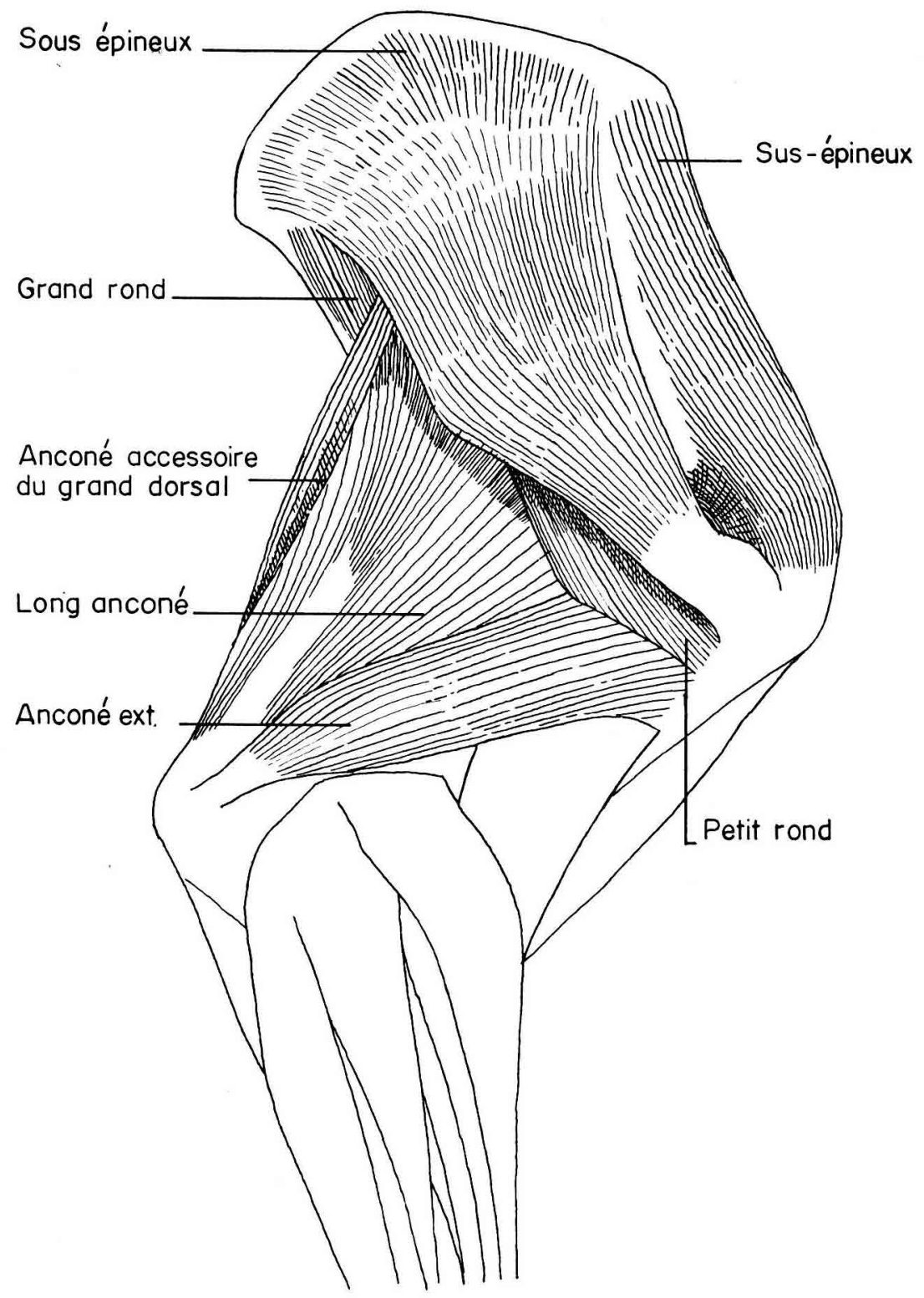

M. B. et D.F 


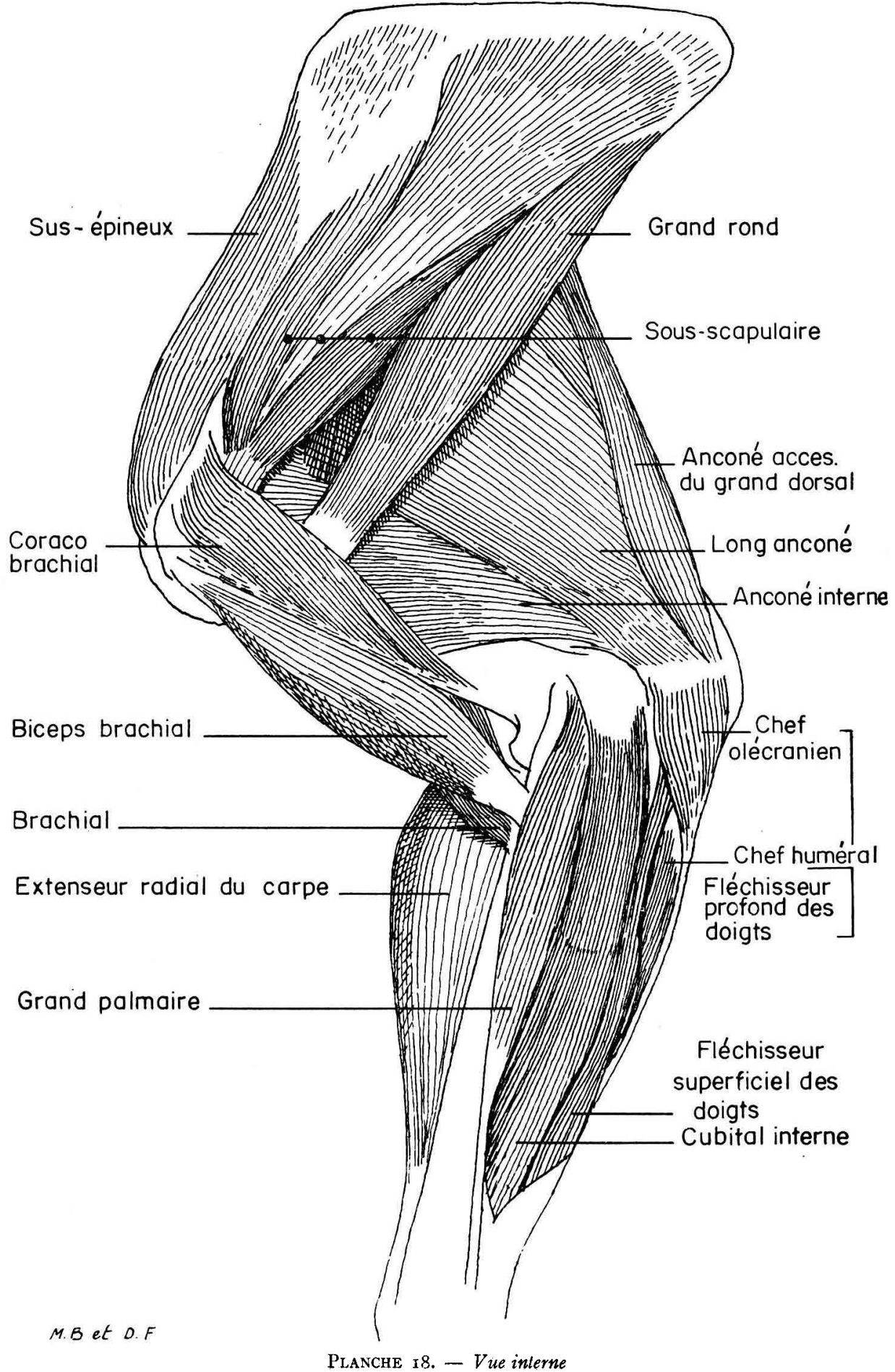




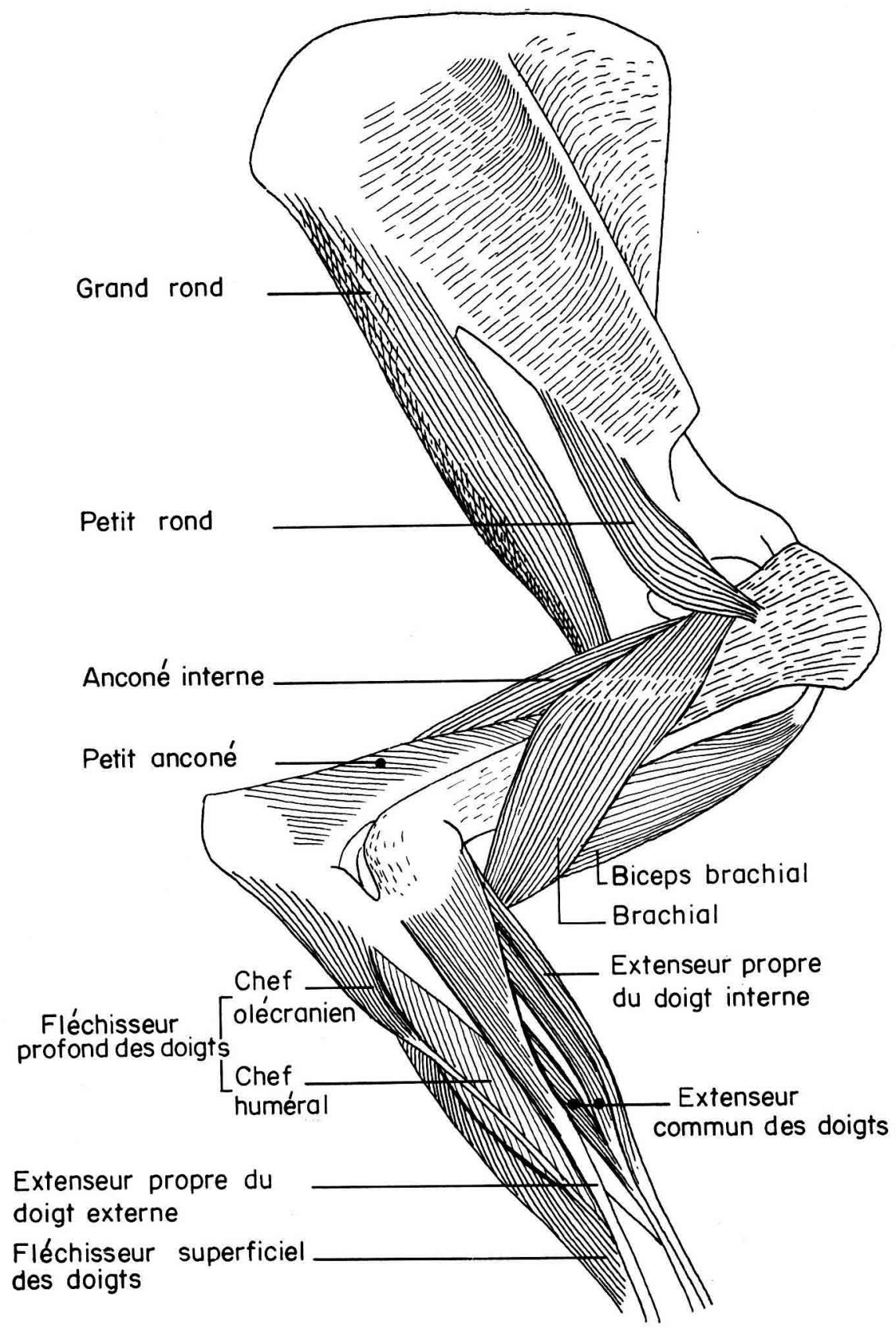




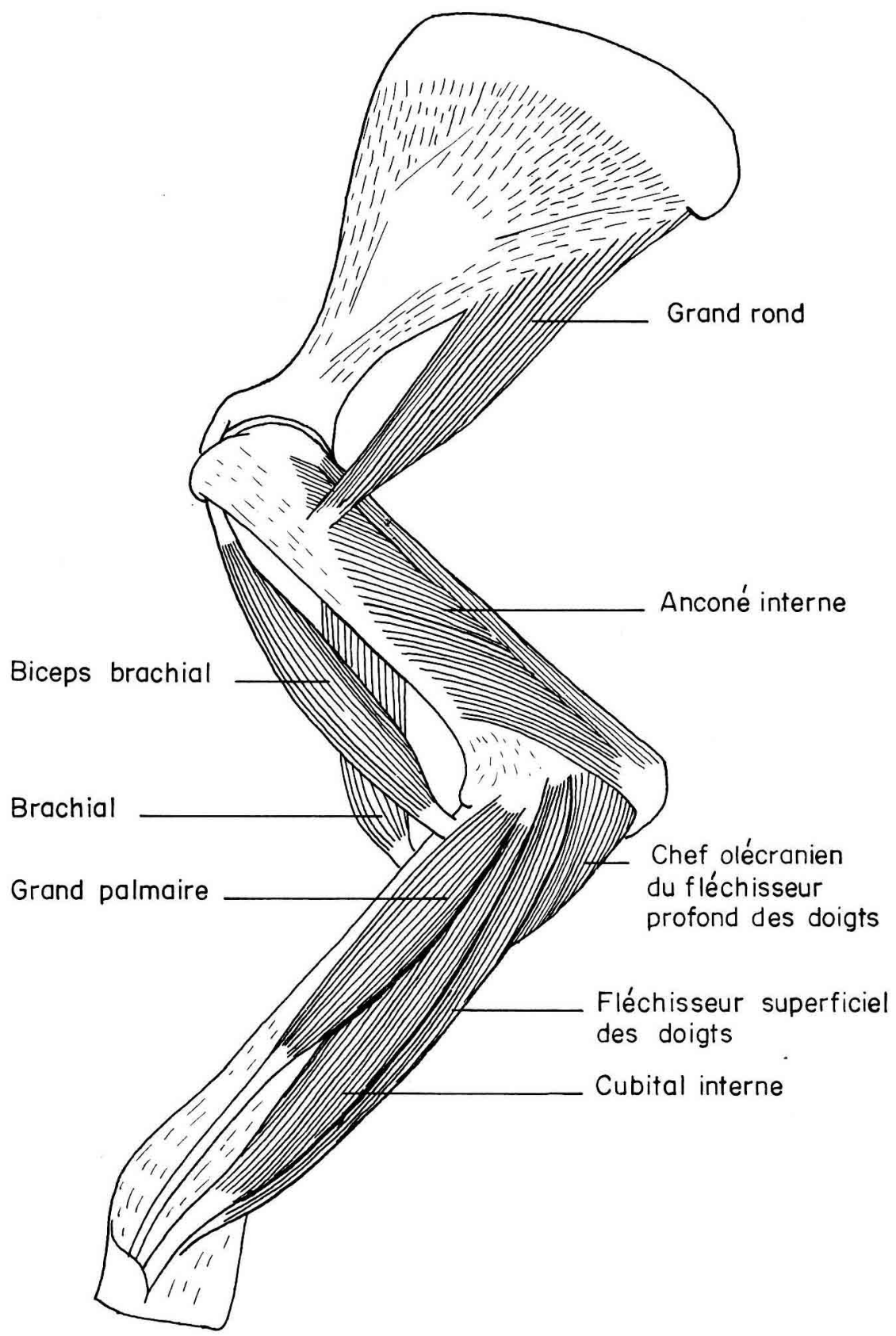

M. A et D.F 


\section{LISTE DES MUSCLES PAR ORDRE ALPHABÉTIQUE}

\begin{tabular}{|c|c|c|}
\hline Nom du muscle & $\begin{array}{c}\text { Numéro } \\
\text { dans le texte }\end{array}$ & Numéro des planches \\
\hline Adducteur de la cuisse $\ldots \ldots \ldots \ldots \ldots \ldots \ldots \ldots$ & 55 & $8,11,12$ \\
\hline Anconé accessoire du grand dorsal ............. & 85 & $16,17,18,1$ \\
\hline 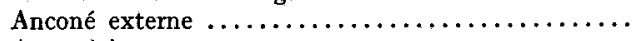 & 87 & $16,17,1$ \\
\hline Anconé interne $\ldots \ldots \ldots \ldots \ldots \ldots \ldots \ldots \ldots$ & 88 & $18,19,20$ \\
\hline 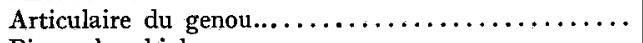 & 66 & 13,15 \\
\hline 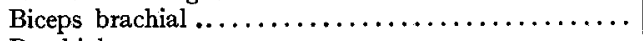 & 90 & $16,18,19,20$ \\
\hline 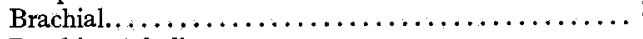 & 91 & $16,18,19,20$ \\
\hline Brachio-céphalique $\ldots \ldots \ldots \ldots \ldots \ldots \ldots \ldots \ldots$ & 2 & 1,2 \\
\hline 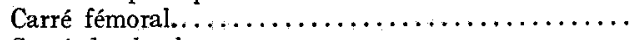 & 60 & $8,12,13,15$ \\
\hline 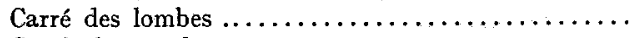 & 46 & 9,10 \\
\hline 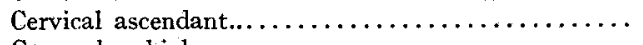 & 16 & 4 \\
\hline Coraco-brachial $\ldots \ldots \ldots \ldots \ldots \ldots \ldots \ldots \ldots \ldots$ & 84 & 18 \\
\hline 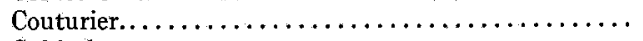 & 49 & 9,10 \\
\hline Cubital externe $\ldots \ldots \ldots \ldots \ldots \ldots \ldots \ldots \ldots \ldots \ldots \ldots \ldots \ldots$ & 96 & 16 \\
\hline Cubital interne $\ldots \ldots \ldots \ldots \ldots \ldots \ldots \ldots \ldots \ldots \ldots \ldots \ldots \ldots \ldots$ & 98 & 18,20 \\
\hline 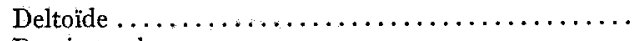 & 78 & $16,1,3$ \\
\hline 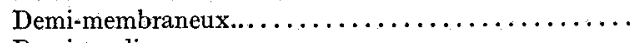 & 53 & $7,8,9,10,11,12$ \\
\hline Demi-tendineux $\ldots \ldots \ldots \ldots \ldots \ldots \ldots \ldots \ldots \ldots \ldots$ & 52 & $7,8,9,10$ \\
\hline Droit antérieur de la cuisse $\ldots \ldots \ldots \ldots \ldots \ldots \ldots$ & 63 & $10,11,12$ \\
\hline 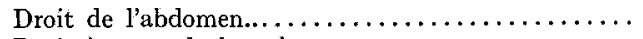 & 41 & $7,8,9,1,3,4$ \\
\hline Droit interne de la cuisse $\ldots \ldots \ldots \ldots \ldots \ldots \ldots$ & 50 & 9,10 \\
\hline Droit latéral de la tête $\ldots \ldots \ldots \ldots \ldots \ldots \ldots \ldots$ & 33 & non représenté \\
\hline Droit du thorax $\ldots \ldots \ldots \ldots \ldots \ldots \ldots \ldots \ldots \ldots$ & 18 & 4 \\
\hline Droit ventral de la tête..$\ldots \ldots \ldots \ldots \ldots \ldots \ldots$ & 32 & non représenté \\
\hline Épineux du cou $\ldots \ldots \ldots \ldots \ldots \ldots \ldots \ldots \ldots \ldots \ldots \ldots \ldots \ldots \ldots \ldots \ldots$ & 24 & 5 \\
\hline Extenseur commun des doigts $\ldots \ldots \ldots \ldots \ldots \ldots$ & 95 & 16,19 \\
\hline Extenseur oblique du métacarpe $\ldots \ldots \ldots \ldots \ldots$ & 101 & 16 \\
\hline Extenseur propre du doigt externe (M. A.)......... & 94 & 16,19 \\
\hline Extenseur propre du doigt externe (M. P.)......... & 72 & 13 \\
\hline Extenseur propre du doigt interne.$\ldots \ldots \ldots \ldots \ldots$ & 93 & 16,19 \\
\hline 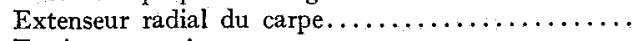 & 92 & 16,18 \\
\hline Fessier accessoire $\ldots \ldots \ldots \ldots \ldots \ldots \ldots \ldots \ldots \ldots$ & 57 & 12 \\
\hline 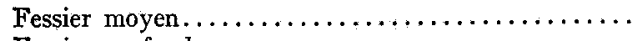 & 56 & 7,8 \\
\hline Fessier profond $\ldots \ldots \ldots \ldots \ldots \ldots \ldots \ldots \ldots \ldots \ldots \ldots \ldots \ldots \ldots \ldots$ & 58 & 13,15 \\
\hline Fléchisseur latéral des doigts.................. & 75 & $13,14,15$ \\
\hline Fléchisseur médial des doigts ................ & 76 & 15 \\
\hline Fléchisseur profond des doigts $\ldots \ldots \ldots \ldots \ldots \ldots$ & 100 & $16,18,19,20$ \\
\hline Fléchisseur superficiel des doigts $\ldots \ldots \ldots \ldots \ldots \ldots$ & 99 & $18,19,20$ \\
\hline Grand complexus $\ldots \ldots \ldots \ldots \ldots \ldots \ldots \ldots \ldots$ & 22 & 4 \\
\hline 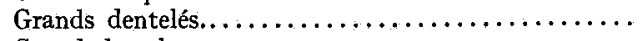 & 14 & 1,3 \\
\hline 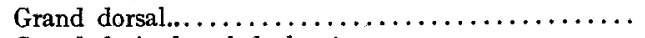 & 12 & 1,7 \\
\hline 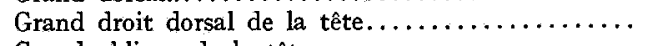 & 29 & 5 \\
\hline 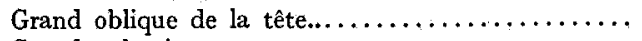 & 28 & 5 \\
\hline Grand palmaire $\ldots \ldots \ldots \ldots \ldots \ldots \ldots \ldots \ldots \ldots$ & 97 & 18,20 \\
\hline 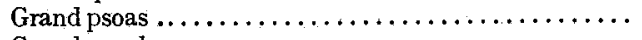 & 45 & 9,10 \\
\hline 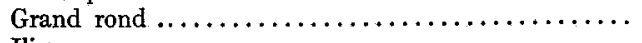 & 83 & $3,16,17,18,19,20$ \\
\hline Iliaque $\ldots \ldots \ldots \ldots \ldots \ldots \ldots \ldots \ldots \ldots \ldots \ldots \ldots \ldots \ldots \ldots \ldots$ & 47 & $8,10,11,12$ \\
\hline Intertransversaire du cou $\ldots \ldots \ldots \ldots \ldots \ldots \ldots$ & 26 & \\
\hline Jumeau du bassin $\ldots \ldots \ldots \ldots \ldots \ldots \ldots \ldots \ldots$ & 59 & $8,12,13$ \\
\hline
\end{tabular}




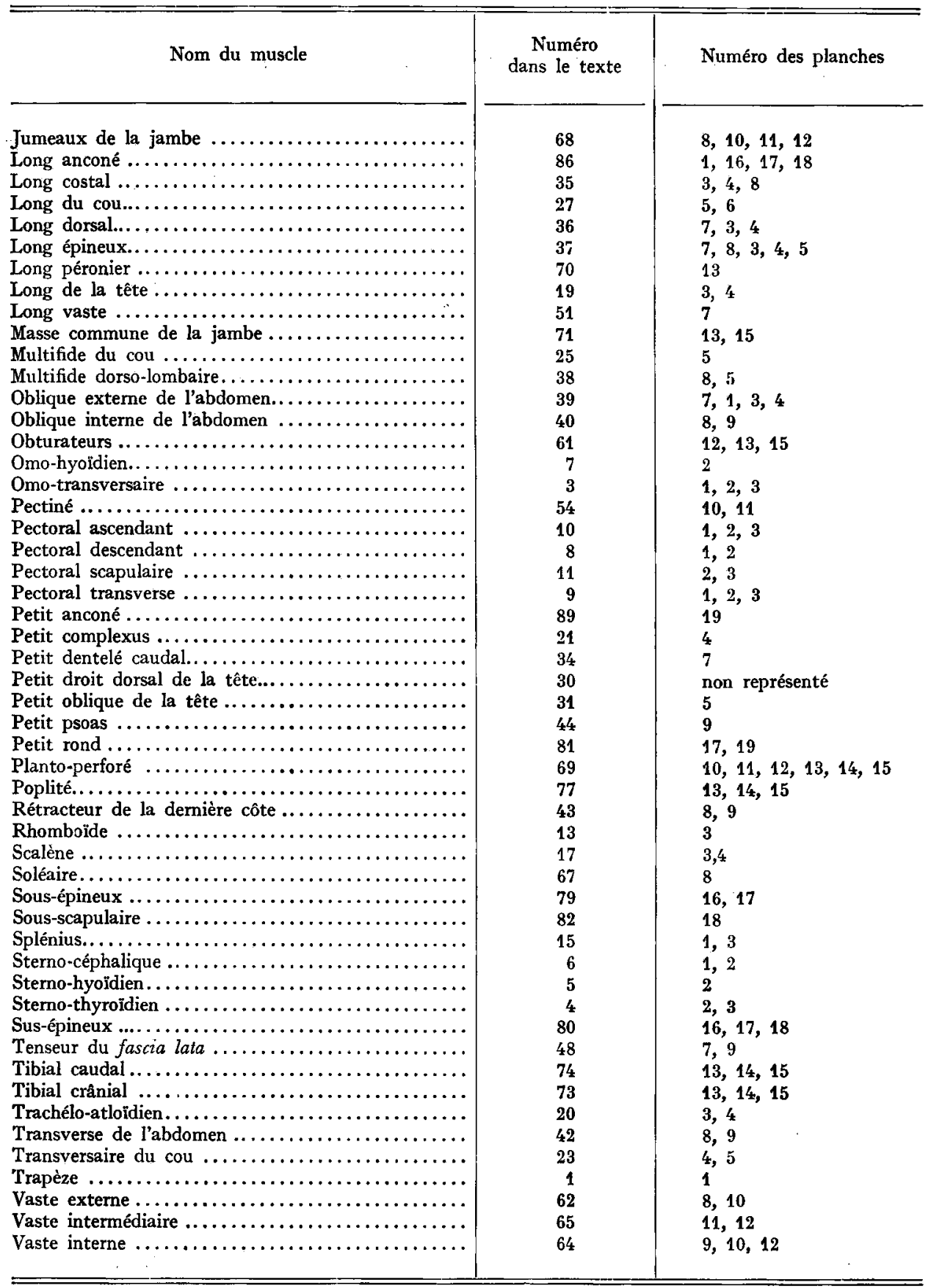




\section{SUMMARY}

THE ANATOMY OF THE MAIN MUSCLES OF THE SHEEP CARCASS

A dissection technique of the sheep carcass is described, in view of further investigations on the growth and development of the sheep.

The main muscles of the carcass and their insertions are successively described according to the pattern of removal, and illustrated in anatomical plates followed by an alphabetical index.

\section{RÉFÉRENCES BIBLIOGRAPHIQUES}

Barone R., I967. Problèmes internationaux de nomenclature en Anatomie. Revue Méd., vét., 118, 749-754, Chauveau A., Arloing S., Lesbre F.-X., 1903. Traité d'anatomie comparée des animaux domestiques* J.-B. Baillière et Fils, Paris, pp. 684 .

CoRnevin, LeSBRE., I892. Caractères miologiques et splanchnologiques différentiels du Mouton et de la Chèvre. Comparaison avec le Chabin. Imprimerie L. Bourgeon, Lyon.

Fourie P.-D., 1962. Growth and development of sheep. I. A carcass dissection technique. $N . Z$. $J l$. agric. Res., 5, 190-222.

May N. D. S., 1964. The Anatomy of the sheep. University of Queensland Press.

Montane L., Bourdelle E., IgI7. Anatomie régionale des animaux domestiques. II. Ruminants. J.-B. Baillière et Fils, Paris, pp. 384 .

Sisson S., Grossman J. D., I953. The anatomy of the domestic animals. W. B. Saunders Company, Philadelphia, pp. 972.

TAGAND R., Barone R., I952. Anatomie des équidés domestiques. École national: vétérinaire de Lyon. Imprimerie Camille Annequin, Lyon, 719-1260. 